\title{
SPLINE APPROXIMATION FOR RETARDED SYSTEMS AND THE RICCATI EQUATION*
}

\section{F. KAPPEL $\dagger$ AND D. SALAMON $\ddagger$}

\begin{abstract}
The purpose of this paper is to introduce a new spline approximation scheme for retarded functional differential equations. The special feature of this approximation scheme is that it preserves the product space structure of retarded systems and approximates the adjoint semigroup in a strong sense. These facts guarantee the convergence of the solution operators for the differential Riccati equation in a strong sense. Numerical findings indicate a significant improvement in the convergence behaviour over both the averaging and the previous spline approximation scheme.
\end{abstract}

Key words. retarded functional differential equations, approximation, splines, Riccati equation

AMS(MOS) subject classifications. 34K35, 41A15, 93D15

1. Introduction. In this paper we introduce a new spline approximation scheme for linear time invariant retarded functional differential equations (RFDEs) and establish a number of convergence results. In particular we show that the approximate feedback law and the solution of the operator Riccati equation, associated with the linear quadratic control problem for this class of systems, converge in the uniform operator topology.

The first step of the general approach is to transform the RFDE

$$
\dot{x}(t)=L x_{t}+B_{0} u(t), \quad y(t)=C_{0} x(t)
$$

into an abstract Cauchy problem of the form

$$
\frac{d}{d t} x(t)=\mathscr{A} x(t)+\mathscr{B} u(t), \quad y(t)=\mathscr{C}_{x}(t)
$$

in the Hilbert space $\mathscr{X}=\mathbb{R}^{n} \times L^{2}\left[-h, 0 ; \mathbb{R}^{n}\right], h>0$, where $\mathscr{A}$ is the infinitesimal generator of the strongly continuous semigroup $\mathscr{S}(t)$ which is associated with the uncontrolled delay equation. For systems of the form (1.2) there exists a general theory of the linear quadratic control problem of minimizing the cost functional

$$
J(u)=\left\langle x(T), \mathscr{G}_{x}(T)\right\rangle+\int_{0}^{T}\left[|y(t)|^{2}+|u(t)|^{2}\right] d t
$$

(see e.g. [9], [14], [19]). The optimal control can be characterized as a feedback law which is determined by an operator satisfying the differential Riccati equation (in the case $T<\infty$ ), respectively, the algebraic Riccati equation (in the case $T=\infty$ ). These operator Riccati equations involve both the original generator $\mathscr{A}$ and its adjoint operator $\mathscr{A}^{*}$. Therefore, in order to approximate the feedback law and the Riccati operator in the strong operator topology, we have to approximate both semigroups $\mathscr{S}(t)$ and $\mathscr{S}^{*}(t)$ in the strong operator topology (see [20]).

* Received by the editors May 3, 1984; accepted for publication (in revised form) June 2, 1986. This work was supported in part by the Fonds zur Förderung der wissenschatlichen Forschung, Austria, under project P4534.

† Institute for Mathematics, University of Graz, Elisabethstrasse 16, A-8010 Graz, Austria. The work of this author was supported in part by the University of Bremen, West Germany, and was begun while the author held a visiting professorship at the Forschungsschwerpunkt Dynamische Systeme, University of Bremen, in October 1982.

¥ Mathematics Research Center, University of Wisconsin, Madison, Wisconsin 53706. The work of this author was supported in part by National Science Foundation grant MCS-8210950. 
For the approximation of the semigroups we use a Galerkin type scheme, i.e., we define finite dimensional subspaces $\mathscr{X}^{N}$ of $\mathscr{X}$ and operators $\mathscr{A}^{N}$ on $\mathscr{X}^{N}$ which generate semigroups $\mathscr{S}^{N}(t)$ on $\mathscr{X}^{N}$. The classical idea is to choose $\mathscr{X}^{N} \subset \operatorname{dom} \mathscr{A}$ and define $\mathscr{A}^{N}=p^{N} \mathscr{A} p^{N}$, where $p^{N}$ is the orthogonal projection of $\mathscr{X}$ onto $\mathscr{X}^{N}$. Under appropriate consistency and stability hypotheses the convergence of $\mathscr{S}^{N}(t)$ to $\mathscr{S}(t)$ in the strong operator topology follows.

These ideas have been used by Banks and Kappel [7] for the development of a spline approximation scheme for RFDEs and have then been applied to problems of optimal control and parameter identification e.g. in [3], [6], [8], [27]. In particular, Kunisch [27] has established weak convergence results for the solution operators of the differential Riccati equations. Numerical findings in [8] indicate that these operators indeed do not converge strongly for the spline scheme developed in [7]. The main reason for this seems to be that the subspace $\mathscr{X}^{N}$ in [7] has been chosen to be contained in the domain of $\mathscr{A}$ which is different from the domain of $\mathscr{A}^{*}$.

In order to overcome this unequal treatment of $\mathscr{S}(t)$ and $\mathscr{S}^{*}(t)$, our idea was to enlarge the subspace $\mathscr{X}^{N}$ such that it is neither contained in dom $\mathscr{A}$ nor in dom $\mathscr{A}^{*}$, but contains sufficiently many elements of both domains. Of course, in this situation the approximating operators can no longer be defined by $\mathscr{A}^{N}=p^{N} \mathscr{A} p^{N}$ but have to be defined directly instead (for details see $\S 5.1$ ). As a result we are able to establish the desired convergence of the solution operators of the Riccati equation in the uniform operator topology for the finite time horizon problem. Despite the fact that in the case of the infinite time horizon problem our scheme always did converge numerically, we were not able to prove this convergence following the approach presented in [20]. The reason is that we do not have the uniform (with respect to $N$ ) exponential stability of the approximating semigroups for our scheme (compare the remarks at the end of $\S 5.3$ ). In this respect the spline approximation scheme differs from the averaging approximation scheme in [4] for which the uniform exponential stability property has been established in [38].

In two preliminary sections we collect some basic facts from the state space and control theory for retarded systems $(\S 2)$ and give a short survey on the theory of the linear quadratic optimal control problem for abstract systems in Hilbert space and for RFDEs ( $\$ 3)$. In $\S 4.1$ we present a general approximation scheme for abstract Cauchy problems in Banach space. In $\$ 4.2$ we consider the problem of approximating the feedback law for the finite time horizon problem following the approach given by Gibson in [20].

The main part of this paper is $\S 5$, where we develop a special spline scheme and prove convergence results along the general ideas given in $\S \S 4.1$ and 4.2. We also give the explicit formulae for the matrices which are necessary for the implementation of our scheme. This scheme has remarkable qualitative properties which will be published elsewhere.

Finally, in $\S 6$ we present some of the many numerical calculations in order to demonstrate the good behaviour of our scheme and the advantage it offers over both the averaging approximation scheme [4], [20] and the spline scheme in [7], [8].

2. State space theory for linear hereditary control systems.

2.1. Linear hereditary control systems. We consider the linear hereditary control system

$$
\begin{aligned}
& \dot{x}(t)=L x_{t}+B_{0} u(t), \quad t \geqq 0, \\
& y(t)=C_{0} x(t),
\end{aligned}
$$


where $x(t) \in \mathbb{R}^{n}, u(t) \in \mathbb{R}^{l}, y(t) \in \mathbb{R}^{m}$ and $x_{t}$ is defined by $x_{t}(s)=x(t+s)$ for $-h \leqq s \leqq 0$, $h>0$. Correspondingly $B_{0}$ and $C_{0}$ are real matrices of appropriate dimensions and $L$ is a bounded linear functional $C\left(-h, 0 ; \mathbb{R}^{n}\right) \rightarrow \mathbb{R}^{n}$ given by

$$
L \phi=\sum_{j=0}^{p} A_{j} \phi\left(-h_{j}\right)+\int_{-h}^{0} A_{01}(\tau) \phi(\tau) d \tau, \quad \phi \in C\left(-h, 0 ; \mathbb{R}^{n}\right),
$$

where $0=h_{0}<\cdots<h_{p}=h$ and $A_{j} \in \mathbb{R}^{n \times n}, j=0, \cdots, p$, as well as $A_{01}(\cdot) \in$ $L^{2}\left(-h, 0 ; \mathbb{R}^{n \times n}\right)$. A solution of $(2.1 \mathrm{a})$ is a function $x(\cdot) \in L_{\mathrm{loc}}^{2}\left(-h, \infty ; \mathbb{R}^{n}\right)$ which is absolutely continuous with $L^{2}$-derivative on every compact interval $[0, T], T>0$, and satisfies (2.1a) for almost all $t \geqq 0$. It is well known that (2.1a) admits a unique solution $x(t)=x(t ; \phi, u)$ for every input $u(\cdot) \in L_{\mathrm{loc}}^{2}\left(0, \infty ; \mathbb{R}^{l}\right)$ and every initial condition

$$
x(0)=\phi^{0}, \quad x(\tau)=\phi^{1}(\tau), \quad-h \leqq \tau<0,
$$

where $\phi=\left(\phi^{0}, \phi^{1}\right) \in M^{2}=\mathbb{R}^{n} \times L^{2}\left(-h, 0 ; \mathbb{R}^{n}\right)$. Moreover, $x(\cdot ; \phi, u)$ depends continuously on $\phi$ and $u$ on compact intervals, i.e., for any $T>0$ there exists a $K>0$ such that

$$
\sup _{0 \leqq t \leqq T}|x(t ; \phi, u)| \leqq K\left(\|\phi\|+\|u\|_{L^{2}\left(0, T ; \mathbb{R}^{\prime}\right)}\right),
$$

where $\|\phi\|=\left(\left|\phi^{0}\right|^{2}+\left|\phi^{1}\right|_{L^{2}}^{2}\right)^{1 / 2}$ for $\phi \in M^{2}$ (see e.g. [12], [18]). The fundamental solution of (2.1a) will be denoted by $X(t)$ and is the $n \times n$ matrix valued solution of (2.1a) which corresponds to $u \equiv 0$ and $X(0)=I, X(\tau)=0$ for $-h \leqq \tau<0$. The Laplace transform of $X(\cdot)$ is given by $\Delta^{-1}(\lambda)$, where

$$
\begin{aligned}
\Delta(\lambda) & =\lambda I-L\left(e^{\lambda \cdot} I\right) \\
& =\lambda I-\sum_{j=0}^{p} A_{j} e^{-\lambda h_{j}}-\int_{-h}^{0} A_{01}(\tau) e^{\lambda \tau} d \tau, \quad \lambda \in \mathbb{C}
\end{aligned}
$$

is the characteristic matrix of (2.1a). Again it is well known that the forced motion of (2.1a) (in case $\phi=0$ ) can be written as

$$
x(t ; 0, u)=\int_{0}^{t} X(t-\tau) B_{0} u(\tau) d \tau, \quad t \geqq 0 .
$$

2.2. Semigroups and state space description. Existence, uniqueness and continuous dependence results for solutions of RFDEs motivate the definition of the state of system (2.1) to be the pair

$$
w(t)=\left(x(t), x_{t}\right) \in M^{2},
$$

which completely describes the past history of the solution at time $t \geqq 0$. The evolution of this state is governed by the variation-of-constants formula

$$
w(t)=S(t) \phi+\int_{0}^{t} S(t-s) B u(s) d s, \quad t \geqq 0,
$$

which is the infinite dimensional version of $(2.3)$. The input operator $B: \mathbb{R}^{l} \rightarrow M^{2}$ is given by

$$
B u=\left(B_{0} u, 0\right) \in M^{2}, \quad u \in \mathbb{R}^{l},
$$

and the semigroup $S(\cdot)$ corresponds to the free motion of the system, i.e., $S(t): M^{2} \rightarrow$ $M^{2}, t \geqq 0$ is defined by

$$
S(t) \phi=\left(x(t ; \phi, 0), x_{t}(\phi, 0)\right), \quad t \geqq 0, \quad \phi \in M^{2} .
$$


The infinitesimal generator of $S(\cdot)$ is given by

$$
\begin{aligned}
& \operatorname{dom} A=\left\{\phi \in M^{2} \mid \phi^{1} \in W^{1,2}, \phi^{0}=\phi^{1}(0)\right\}, \\
& A \phi:=\left(L \phi^{1}, \dot{\phi}^{1}\right),
\end{aligned}
$$

where $W^{1,2}$ denotes the Sobolev space $W^{1,2}\left(-h, 0 ; \mathbb{R}^{n}\right)$. The function $w(t)$ as defined in (2.5) is a mild solution of the abstract system

$$
\begin{aligned}
& \dot{w}(t)=A w(t)+B u(t), \\
& y(t)=C w(t), \quad w(0)=\phi .
\end{aligned}
$$

The output operator $C: M^{2} \rightarrow \mathbb{R}^{m}$ is defined by $C \phi=C_{0} \phi^{0}, \phi \in M^{2}$.

The operator $A^{*}$ dual to $A$ is explicitly given by (see e.g. [17]):

$$
\begin{aligned}
& \operatorname{dom} A^{*}=\left\{f \in M^{2} \mid f^{1}+\sum_{j=1}^{p-1} A_{j}^{T} f^{0} \chi_{\left[-h,-h_{j}\right]} \in W^{1,2}, f^{1}(-h)=A_{p}^{T} f^{0}\right\}, \\
& {\left[A^{*} f\right]^{0}=f^{1}(0)+A_{0}^{T} f^{0},} \\
& {\left[A^{*} f\right]^{1}(\tau)=A_{01}^{T}(\tau) f^{0}-\frac{d}{d \tau}\left[f^{1}(\tau)+\sum_{j=1}^{p-1} A_{j}^{T} f^{0} \chi_{\left[-h,-h_{j}\right]}(\tau)\right] .}
\end{aligned}
$$

The characteristic function of an interval $I$ is denoted by $\chi_{I}$.

2.3. Stability, stabilizability and controllability. System (2.1) is said to be stable if every solution $x(t)$ of the free system (i.e. $u(t) \equiv 0)$ tends to zero as $t$ goes to infinity. Equivalently, the semigroup $S(\cdot)$ is exponentially stable, i.e.,

$$
\omega_{0}=\lim _{t \rightarrow \infty} \frac{1}{t} \ln \|S(t)\|=\sup \{\operatorname{Re} \lambda \mid \lambda \in \sigma(A)\}<0
$$

(see for instance [22]). The spectrum of $A$ is given by $\sigma(A)=\{\lambda \in \mathbb{C} \mid \operatorname{det} \Delta(\lambda)=0\}$. Note that $\sigma\left(A^{*}\right)=\sigma(A)$.

The control system (2.1) is said to be stabilizable if there exists a control law

$$
\begin{aligned}
u(t) & =K\left(x(t), x_{t}\right) \\
& =K_{0} x(t)+\int_{-h}^{0} K_{1}(\tau) x(t+\tau) d \tau,
\end{aligned}
$$

where $K_{0} \in \mathbb{R}^{l \times n}, K_{1}(\cdot) \in L^{2}\left(-h, 0 ; \mathbb{R}^{l \times n}\right)$, such that the closed loop system (2.1), (2.8) is stable. We have the following important characterization (see [33], [36]).

THEOREM 2.1. The following statements are equivalent:

(i) System (2.1) is stabilizable.

(ii) There exists a $K \in \mathscr{L}\left(M^{2}, \mathbb{R}^{l}\right)$ such that the operator $A+B K$ generates an exponentially stable $C_{0}$-semigroup.

(iii) $\operatorname{rank}\left[\Delta(\lambda), B_{0}\right]=n$ for all $\lambda \in \mathbb{C}$ with $\operatorname{Re} \lambda \geqq 0$.

The dual result is the following (see e.g. [10] or [36], [37]):

THEOREM 2.2. The following statements are equivalent:

(i) There exists a $H \in \mathscr{L}\left(\mathbb{R}^{m}, M^{2}\right)$ such that the operator $A+H C$ generates an exponentially stable semigroup.

(ii) rank $\left(\begin{array}{c}\Delta(\lambda) \\ C_{0}\end{array}\right)=n$ for all $\lambda \in \mathbb{C}$ with $\operatorname{Re} \lambda \geqq 0$.

System (2.1) is called detectable if the statements of the previous theorem are satisfied. A detailed discussion of the duality relations between feedback stabilization and dynamic observation in the product space framework can be found in [36]. 
3. The linear quadratic control problem.

3.1. Control systems in Hilbert spaces. Let us first deal with general linear control systems in Hilbert spaces $\mathscr{X}, \mathcal{U}$ and $\mathscr{Y}$ described by

$$
\begin{aligned}
& \dot{x}(t)=\mathscr{A} x(t)+\mathscr{B} u(t), \quad x(0)=x_{0}, \\
& y(t)=\mathscr{C} x(t) .
\end{aligned}
$$

We assume that $\mathscr{B} \in \mathscr{L}(\mathscr{U}, \mathscr{X}), \mathscr{C} \in \mathscr{L}(\mathscr{X}, \mathscr{Y})$ and that $\mathscr{A}$ is the infinitesimal generator of a $C_{0}$-semigroup $\mathscr{S}(t)$ on $\mathscr{X}$. System (3.1) will be understood in the sense of mild solutions, i.e., the trajectories of the system are given by

$$
x(t)=\mathscr{S}(t) x_{0}+\int_{0}^{t} \mathscr{S}(t-s) \mathscr{B} u(s) d s, \quad t \geqq 0
$$

for any $x_{0} \in \mathscr{X}$ and any input $u(\cdot) \in L_{\text {loc }}^{2}(0, \infty ; \mathscr{U})$.

Let $\mathscr{R}: \mathscr{U} \rightarrow \mathscr{U}$ and $\mathscr{G}: \mathscr{X} \rightarrow \mathscr{X}$ be selfadjoint linear operators satisfying

$$
\left\langle x, \mathscr{G}_{x}\right\rangle \geqq 0 \text { for all } x \in \mathscr{X}
$$

and

$$
\langle u, \mathscr{R} u\rangle \geqq \varepsilon\|u\|^{2} \quad \text { for all } u \in \mathscr{U}
$$

with some $\varepsilon>0$. In this section we look at the control problem of minimizing the cost functional

$$
J(u)=\left\langle x(T), \mathscr{C}_{x}(T)\right\rangle+\int_{0}^{T}\left[\left\|\mathscr{C}_{x}(t)\right\|^{2}+\langle u(t), \mathscr{R} u(t)\rangle\right] d t,
$$

where $x(t)$ is given by (3.2) and $T>0$ is a fixed final time. For the proof of the following result see [14] and [19].

THEOREM 3.1. For any $x_{0} \in \mathscr{X}$ there exists a unique control function $\bar{u}(\cdot) \in$ $L^{2}(0, T ; \mathcal{U})$ which minimizes the cost functional (3.3) under the constraint (3.2). The optimal control is of feedback form and is given by

$$
\bar{u}(t)=-\mathscr{R}^{-1} \mathscr{B}^{*} \mathscr{P}(t) \bar{x}(t), \quad t \geqq 0,
$$

where $\bar{x}(t)$ is the mild solution of the Cauchy problem $\dot{x}=\left(\mathscr{A}-\mathscr{B}^{-1} \mathscr{B} * \mathscr{P}(t)\right) x, x(0)=$ $x_{0}$, and $t \rightarrow \mathscr{P}(t) \in \mathscr{L}(\mathscr{X})$ is the unique operator valued function on $[0, T]$ with the following properties:

(i) $\mathscr{P}(t)$ is positive semidefinite for every $t \in[0, T]$.

(ii) The function $t \rightarrow \mathscr{P}(t)_{x}$ is continuous on $[0, T]$ for every $x \in \mathscr{X}$, and satisfies the Riccati integral equation

$$
\begin{aligned}
\mathscr{P}(t)_{x}=\mathscr{S}^{*}(T-t) \mathscr{G}(T-t) x & \\
+\int_{t}^{T} \mathscr{S}^{*}(\tau-t)\left[\mathscr{C}^{*} \mathscr{C}-\mathscr{P}(\tau) \mathscr{B} \mathscr{R}^{-1} \mathscr{B}^{*} \mathscr{P}(\tau)\right] \mathscr{S}(\tau-t) x d \tau, & 0 \leqq t \leqq T, \quad x \in \mathscr{X} .
\end{aligned}
$$

Moreover, the optimal cost is given by

$$
J(\bar{u})=\left\langle x_{0}, \mathscr{P}(0) x_{0}\right\rangle .
$$

In [19] it is also shown that $\mathscr{P}(t)$ satisfies

$$
\mathscr{P}(t) x=\mathscr{S}^{*}(T-t) \mathscr{G} \Phi(T, t) x+\int_{t}^{T} \mathscr{S}^{*}(\tau-t) \mathscr{C}^{*} \mathscr{C} \Phi(\tau, t) x d t, \quad 0 \leqq t \leqq T, \quad x \in \mathscr{X}
$$


where $\Phi(\tau, t)$ is the evolution operator given by

$$
\begin{array}{r}
\Phi(\tau, t) x=\mathscr{P}(\tau-t) x-\int_{t}^{\tau} \mathscr{S}(\tau-\sigma) \mathscr{B}^{-1} \mathscr{B} * \mathscr{P}(\sigma) \Phi(\sigma, t) x d \sigma, \\
0 \leqq t \leqq \tau \leqq T, \quad x \in \mathscr{X} .
\end{array}
$$

Let us now consider the problem of minimizing the cost functional

$$
J(u)=\int_{0}^{\infty}\left[\left\|\mathscr{C}_{x}(t)\right\|^{2}+\langle u(t), \mathscr{R} u(t)\rangle\right] d t,
$$

where again $x(t)$ is given by (3.2). For this situation the following result has been proved (see [14], [15], [41]; further references can be found in the survey paper [9]):

THEOREM 3.2. (a) The following statements are equivalent:

(i) For any $x_{0} \in \mathscr{X}$ there exists an input $u(\cdot) \in L^{2}(0, \infty ; \mathscr{U})$ such that the corresponding cost $J(u)$ given by (3.8) and (3.2) is finite.

(ii) There exists a positive semidefinite operator $\mathscr{P} \in \mathscr{L}(\mathscr{X})$ satisfying the algebraic Riccati operator equation

$$
\left\langle\mathscr{A}_{y}, \mathscr{P}_{x}\right\rangle+\left\langle\mathscr{P}_{y}, \mathscr{A}_{x}\right\rangle+\left\langle\mathscr{C}_{y}, \mathscr{C}_{x}\right\rangle-\left\langle\mathscr{P}_{y}, \mathscr{B}^{-1} \mathscr{B}^{*} \mathscr{P}_{x}\right\rangle=0
$$

for all $x, y \in \operatorname{dom} \mathscr{A}$.

(b) If the statements under (a) are valid, then there exists a unique optimal control $\bar{u}(t)$ which is given by the feedback law

$$
\bar{u}(t)=-\mathscr{R}^{-1} \mathscr{B}^{*} \mathscr{P} \bar{x}(t), \quad t \geqq 0,
$$

where $\bar{x}(t)$ is the mild solution of the Cauchy problem $\dot{x}=\left(\mathscr{A}-\mathscr{B} \mathscr{R}^{-1} \mathscr{B} * \mathscr{P}\right) x, x(0)=x_{0}$, and $\mathscr{P}$ is the minimal solution of (3.9). Moreover, the optimal cost is given by

$$
J(\bar{u})=\left\langle x_{0}, \mathscr{P}_{x_{0}}\right\rangle .
$$

(c) Suppose that the statements under (a) are satisfied and let $\mathscr{P}$ be the minimal positive semidefinite solution of (3.9). Moreover, let $\mathscr{P}_{T}(t), 0 \leqq t \leqq T$, be the unique positive semidefinite solution of (3.5) with $\mathscr{G}=0$. Then $\mathscr{P}$ is the strong limit of $\mathscr{P}_{T}(0)$ as $T$ goes to infinity.

(d) Suppose that there exists some $\mathscr{H} \in L(\mathscr{Y}, \mathscr{X})$ such that the operator $\mathscr{A}+\mathscr{H} \mathscr{C}$ generates an exponentially stable semigroup. Then there exists at most one positive semidefinite solution of (3.9). Moreover, if such a solution exists, then the closed loop semigroup generated by $\mathscr{A}-\mathscr{B} \mathscr{R}^{-1} \mathscr{B} * \mathscr{P}$ is exponentially stable.

3.2. Applications to hereditary systems. Let us first apply Theorem 3.1 to system $(\Sigma)$ which is associated to system (2.1) in terms of the state concept introduced in $\S 2$. The cost functional for system $(2.1)$ is assumed to be

$$
\begin{aligned}
& J(u)=x(T ; \phi, u)^{T} G_{0} x(T ; \phi, u) \\
& \quad+\int_{0}^{T}\left[\left\|C_{0} x(t ; \phi, u)\right\|^{2}+u(t)^{T} R u(t)\right] d t,
\end{aligned}
$$

where $R \in \mathbb{R}^{l \times l}$ is positive definite and $G_{0} \in \mathbb{R}^{n \times n}$ is positive semidefinite. If the operator $G: M^{2} \rightarrow M^{2}$ is defined by $G \phi=\left(G_{0} \phi^{0}, 0\right), \phi \in M^{2}$, then the cost functional for system $(\Sigma)$ is given by (3.3) (with $\mathscr{G}=G, \mathscr{C}=C$ and $\mathscr{R}=R$, of course). According 
to Theorem 3.1 there exists a unique, positive semidefinite, strongly continuous family $\Pi(\cdot)$ of operators in $\mathscr{L}\left(M^{2}\right)$ which satisfies the Riccati integral equation

$$
\begin{aligned}
& \Pi(t) \phi=S^{*}(T-t) G S(T-t) \phi \\
& +\int_{t}^{T} S^{*}(\tau-t)\left[C^{*} C-\Pi(\tau) B R^{-1} B^{*} \Pi(\tau)\right] S(\tau-t) \phi d \tau, \\
& \quad \phi \in M^{2}, \quad 0 \leqq t \leqq T .
\end{aligned}
$$

Let us now look at the structure of the operator $\Pi(t)$. Due to the product space structure of the state space $M^{2}$ we can write

$$
\Pi(t)=\left(\begin{array}{ll}
\Pi_{00}(t) & \Pi_{01}(t) \\
\Pi_{10}(t) & \Pi_{11}(t)
\end{array}\right),
$$

where $\Pi_{00}(t)$ is a selfadjoint operator $\mathbb{R}^{n} \rightarrow \mathbb{R}^{n}$ which can be represented by a symmetric matrix and $\Pi_{11}(t)$ is a selfadjoint operator $L^{2} \rightarrow L^{2}$. The operator $\Pi_{10}(t)$ can be represented by a matrix-valued function $\Pi_{10}(t, \cdot) \in L^{2}\left(-h, 0 ; \mathbb{R}^{n \times n}\right)$. The adjoint operator $\Pi_{01}(t)=\Pi_{10}^{*}(t)$ from $L^{2} \rightarrow \mathbb{R}^{n}$ is given by

$$
\Pi_{01}(t) \phi=\int_{-h}^{0} \Pi_{10}^{T}(t, \tau) \phi(\tau) d \tau, \quad \phi \in L^{2} .
$$

We are mainly interested in the matrices $\Pi_{00}(t)$ and $\Pi_{10}(t, \tau)$, which determine the optimal feedback law

$$
\bar{u}(t)=-R^{-1} B_{0}^{T}\left[\Pi_{00}(t) x(t)+\int_{-h}^{0} \Pi_{10}^{T}(t, \tau) x(t+\tau) d \tau\right]
$$

for system (2.1). Recall that $B^{*}$ maps $\phi \in M^{2}$ to $B_{0}^{T} \phi^{0} \in \mathbb{R}^{l}$.

For the rest of this section we assume that system (2.1) is stabilizable and detectable, so that system $(\Sigma)$ satisfies the assumptions of Theorem 3.2. Hence there exists a positive semi-definite operator $\Pi \in \mathscr{L}\left(M^{2}\right)$ satisfying the algebraic Riccati equation

$$
A^{*} \Pi \phi+\Pi A \phi-\Pi B R^{-1} B^{*} \Pi \phi+C^{*} C \phi=0,
$$

$\phi \in \operatorname{dom} A$. The equation can be written in this form since every solution $\mathscr{P}$ of (3.9) maps dom $\mathscr{A}$ into dom $\mathscr{A}^{*}$, i.e.,

$$
\text { range } \Pi \subset \operatorname{dom} A^{*} \text {. }
$$

Again the operator $\Pi$ can be written in block form

$$
\Pi=\left(\begin{array}{ll}
\Pi_{00} & \Pi_{01} \\
\Pi_{10} & \Pi_{11}
\end{array}\right),
$$

where $\Pi_{01}=\Pi_{10}^{*}$ maps $L^{2}$ into $\mathbb{R}^{n}$. Hence the optimal feedback law is of the form

$$
\begin{aligned}
u(t) & =-R^{-1} B^{*} \Pi\left(x(t), x_{t}\right) \\
& =-R^{-1} B_{0}^{T}\left[\Pi_{00} x(t)+\int_{-h}^{0} \Pi_{10}^{T}(\tau) x(t+\tau) d \tau\right] .
\end{aligned}
$$

Finally note that the closed loop system (2.1), (3.16) is stable (Theorem 3.2).

4. A general approximation scheme.

4.1. Approximation of the state. In this section we present a general approximation scheme for linear abstract Cauchy problems restricting ourselves to a situation which is of sufficient generality for our purposes. 
Let $\mathscr{X}$ be a real Hilbert space with inner product $\langle\cdot, \cdot\rangle$ and corresponding norm $\|\cdot\|$. Furthermore let $\mathscr{A}$ be the infinitesimal generator of the $C_{0}$-semigroup $\mathscr{S}(t), t \geqq 0$, on $\mathscr{X}$. It is a fundamental result that there exists constants $M \geqq 1$ and $\omega \in \mathbb{R}$ such that

$$
\|\mathscr{S}(t)\| \leqq M e^{\omega t}, \quad t \geqq 0 .
$$

In order to approximate the trajectories $\mathscr{S}(t) x_{0}, x_{0} \in \mathscr{X}$, it is a standard idea to choose a sequence $\left\{\mathscr{X}^{N}\right\}$ of finite dimensional subspaces of $\mathscr{X}$ with corresponding orthogonal projections

$$
p^{N}: \mathscr{X} \rightarrow \mathscr{X}^{N}, \quad N=1,2, \cdots,
$$

and to define (in an appropriate way) a sequence $\left\{\mathscr{A}^{N}\right\}$ of linear operators

$$
\mathscr{A}^{N}: \mathscr{X}^{N} \rightarrow \mathscr{X}^{N}, \quad N=1,2, \cdots .
$$

With $\mathscr{A}^{N}$ and $x_{0} \in \mathscr{X}$ we associate the Cauchy problem

$$
\begin{aligned}
& \dot{x}^{N}(t)=\mathscr{A}^{N} x^{N}(t), \quad t \geqq 0, \\
& x^{N}(0)=p^{N} x_{0}
\end{aligned}
$$

on $\mathscr{X}^{N}$. We extend the definition of $\mathscr{A}^{N}$ to all of $\mathscr{X}$ by $\mathscr{A}^{N} x=\mathscr{A}^{N} p^{N} x$ and define the $C_{0}$-semigroup $\mathscr{S}^{N}(t), t \geqq 0$, on $\mathscr{X}$ by

$$
\mathscr{S}^{N}(t) x_{0}=e^{\mathscr{A}^{N} t} x_{0}=e^{\mathscr{A}^{N} t} p^{N} x_{0}+x_{0}-p^{N} x_{0}, \quad t \geqq 0, \quad x_{0} \in \mathscr{X} .
$$

The following hypotheses will be used in order to guarantee the desired convergence $\mathscr{S}^{N}(t) p^{N} x_{0} \rightarrow \mathscr{S}(t) x_{0}$ :

(H1) $\lim _{N \rightarrow \infty} p^{N} x=x$ for all $x \in \mathscr{X}$.

(H2) There exists constants $\tilde{M} \geqq 1$ and $\tilde{\omega} \in \mathbb{R}$ such that

$$
\left\|\mathscr{S}^{N}(t) x\right\| \leqq \tilde{M} e^{\tilde{\omega} t}\|x\|
$$

for all $t \geqq 0, x \in \mathscr{X}^{N}$ and $N=1,2, \cdots$.

(H3) There exists a dense subset $D \subset \operatorname{dom} \mathscr{A}$ which is invariant with respect to $\mathscr{S}(t), t \geqq 0$, such that

(i) $\lim _{N \rightarrow \infty} \mathscr{A}^{N} p^{N} x=\mathscr{A} x$ for all $x \in D$, and

(ii) for any $x \in D$ there exists a function $m(\cdot, x) \in L_{\text {loc }}^{1}(0, \infty ; \mathbb{R})$ such that

$$
\left\|\mathscr{A}^{N} p^{N} \mathscr{S}(t) x\right\| \leqq m(t ; x) \quad \text { a.e. on }[0, \infty)
$$

for all $N$.

Hypothesis ( $\mathrm{H} 2)$ is equivalent to

$\left(\mathrm{H} 2^{*}\right)$ For any $N$ there exists a norm $\|\cdot\|_{N}$ on $\mathscr{X}^{N}$ such that

(i) For some constant $\tilde{M} \geqq 1$

$$
\|x\| \leqq\|x\|_{N} \leqq \tilde{M}\|x\|, \quad x \in \mathscr{X}^{N}, \quad N=1,2, \cdots, \quad \text { and }
$$

(ii) for some constant $\tilde{\omega} \in \mathbb{R}$ all operators $\mathscr{A}^{N}-\tilde{\omega} I$ are dissipative on $\left(\mathscr{X}^{N},\|\cdot\|_{N}\right)$ (i.e. $\left\|\left(\mathscr{A}^{N}-\mu I\right) x\right\|_{N} \geqq(\mu-\tilde{\omega})\left\|_{x}\right\|_{N}$ for $x \in \mathscr{X}^{N}, \mu>\tilde{\omega}$ or, in case $\left(\mathscr{X}^{N},\|\cdot\|_{N}\right)$ is a Hilbert space with inner product $\langle\cdot, \cdot\rangle_{N}$, $\left\langle\mathscr{A}^{N} x, x\right\rangle_{N} \leqq \tilde{\omega}\|x\|_{N}^{2}$ for $x \in \mathscr{X}^{N}$; cf. [32, Thm. 4.2], [30, p. 244]).

The equivalence of $(\mathrm{H} 2)$ and $(\mathrm{H} 2 *)$ follows from the well-known relation between exponential estimates for a semigroup and dissipativity properties of its generator (cf., for instance, [32, Thm. 4.3]). 
THEOREM 4.1. Let (H1)-(H3) be satisfied for the sequences $\mathscr{X}^{N}, p^{N}, \mathscr{A}^{N}, N=1$, $2, \cdots$. Then for all $x_{0} \in \mathscr{X}$

$$
\lim _{N \rightarrow \infty} e^{\mathscr{A}^{N} t} p^{N} x_{0}=\mathscr{S}(t) x_{0}
$$

uniformly for $t$ in bounded intervals.

Proof. If $x_{0} \in \operatorname{dom} \mathscr{A}$ then $d / d t \mathscr{S}(t) x_{0}=\mathscr{A} \mathscr{S}(t) x_{0}$. This together with (4.1) implies

$$
\mathscr{S}(t) x_{0}-\mathscr{S}^{N}(t) p^{N} x_{0}=\mathscr{S}^{N}(t)\left[x_{0}-p^{N} x_{0}\right]+\int_{0}^{t} \mathscr{S}^{N}(t-\tau)\left(\mathscr{A}-\mathscr{A}^{N}\right) \mathscr{S}(\tau) x_{0} d \tau,
$$

$x_{0} \in \operatorname{dom} \mathscr{A}, 0 \leqq t \leqq T$. Let $x_{0} \in D$. Using (H2), we immediately get

$$
\left\|\mathscr{S}(t) x_{0}-\mathscr{S}^{N}(t) p^{N} x_{0}\right\| \leqq \tilde{M} e^{\tilde{\omega} T}\left\{\left\|x_{0}-p^{N} x_{0}\right\|+\int_{0}^{T}\left\|\left(\mathscr{A}-\mathscr{A}^{N}\right) \mathscr{S}(\tau) x_{0}\right\| d \tau\right\}
$$

for $0 \leqq t \leqq T$. Then (4.2) follows from (H1), (H3) and Lebesgue's dominated convergence theorem. Note that $\mathscr{S}(\tau) x_{0} \in D$ for $\tau \in[0, T]$. A density argument using (H2) completes the proof.

The methods in the proof of the previous theorem are well known in connection with numerical approximation of partial differential equations (see for instance the proof of the Lax-Richtmyer equivalence theorem in [21]). For delay equations this approach appears for the first time in [3], [6] and has later on been used in [24]. We equally well could have used the Trotter-Kato theorem [32].

Next we consider the nonhomogeneous problem

$$
\begin{aligned}
& \dot{x}(t)=\mathscr{A} x(t)+\mathscr{B} u(t), \quad t \geqq s, \\
& x(s)=x_{0} \in \mathscr{X},
\end{aligned}
$$

where $u \in L_{\text {loc }}^{2}\left(s, \infty ; \mathbb{R}^{l}\right)$ and $\mathscr{B}$ is a linear operator $\mathbb{R}^{l} \rightarrow \mathscr{X}$. The unique mild solution $x(t)=x\left(t ; s, x_{0}, u\right)$ of $(4.3)$ is given by

$$
x(t)=\mathscr{S}(t-s) x_{0}+\int_{s}^{t} \mathscr{S}(t-\tau) \mathscr{B} u(\tau) d \tau, \quad t \geqq s .
$$

In addition to the approximating sequence $\mathscr{X}^{N}, p^{N}, \mathscr{A}^{N}, N=1,2, \cdots$, introduced above let us assume that $\mathscr{B}^{N}, N=1,2, \cdots$, is a sequence of corresponding input operators $\mathbb{R}^{l} \rightarrow \mathscr{X}^{N}$. Then we consider the approximating systems

$$
\begin{aligned}
& \dot{x}^{N}(t)=\mathscr{A}^{N} x^{N}(t)+\mathscr{B}^{N} u(t), \quad t \geqq s, \\
& x^{N}(s)=p^{N} x_{0}, \quad x_{0} \in \mathscr{X},
\end{aligned}
$$

on $\mathscr{X}^{N}$ with the unique solution $x^{N}(t)=x^{N}\left(t ; s, p^{N} x_{0}, u\right)$ given by

$$
x^{N}(t)=\mathscr{S}^{N}(t-s) p^{N} x_{0}+\int_{s}^{t} \mathscr{S}^{N}(t-\tau) \mathscr{B}^{N} u(\tau) d \tau, \quad t \geqq s,
$$

where $\mathscr{S}^{N}(t): \mathscr{X} \rightarrow \mathscr{X}$ is defined as above.

TheOREM 4.2. Assume that $\mathscr{S}^{N}(\cdot), N=1,2, \cdots$, and $\mathscr{S}(\cdot)$ are $C_{0}$-semigroups on $\mathscr{X}$ such that for constants $M \geqq 1, \omega \in \mathbb{R}$

$$
\left\|\mathscr{S}^{N}(t)\right\| \leqq M e^{\omega t}, \quad t \geqq 0, \quad N=1,2, \cdots,
$$

and for all $x_{0} \in \mathscr{X}$

$$
\lim _{N \rightarrow \infty} \mathscr{S}^{N}(t) p^{N} x_{0}=\mathscr{S}(t) x_{0}
$$


uniformly on bounded t-intervals. Furthermore assume that

$$
\lim _{N \rightarrow \infty} \mathscr{B}^{N} \xi=\mathscr{B} \xi \text { for all } \xi \in \mathbb{R}^{l} \text {. }
$$

Then for all $x_{0} \in \mathscr{X}, T>0$ and $\gamma>0$

$$
\lim _{N \rightarrow \infty} x^{N}\left(t ; s, p^{N} x_{0}, u\right)=x\left(t ; s, x_{0}, u\right)
$$

uniformly for $0 \leqq s \leqq t \leqq T$ and for $u \in L^{2}\left(s, T ; \mathbb{R}^{l}\right)$ with $\|u\|_{L^{2}\left(s, T, \mathbb{R}^{l}\right)} \leqq \gamma$.

We omit the proof of this result since it is only a slight modification of that given in [4] for a very similar result.

It is clear that in this section without any changes $\mathscr{X}$ could have been a real Banach space.

4.2. Approximation of the feedback law in the optimal problem. We restrict ourselves to the finite time control problem of minimizing the cost functional

$$
J_{s}(u)=\left\langle x(T), \mathscr{G}_{x}(T)\right\rangle+\int_{s}^{T}\left[\left\|\mathscr{C}_{x}(t)\right\|^{2}+u(t)^{T} \mathscr{R} u(t)\right] d t
$$

associated with the Cauchy problem (4.3). We assume that the operators $\mathscr{G}: \mathscr{X} \rightarrow \mathscr{X}$, $\mathscr{R}: \mathbb{R}^{l} \rightarrow \mathbb{R}^{l}, \mathscr{C}: \mathscr{X} \rightarrow \mathbb{R}^{m}$ are defined as in $\S 3.1$. As we have seen in that section (with obvious modifications for the case when the initial time $s$ is not necessarily zero), the unique solution of this problem is given by the feedback law

$$
\bar{u}_{s}(t)=-\mathscr{R}^{-1} \mathscr{B} * \mathscr{P}(t) \Phi(t, s) x_{0}, \quad s \leqq t \leqq T,
$$

where $\mathscr{P}(t): \mathscr{X} \rightarrow \mathscr{X}$ is the unique positive semidefinite solution of the Riccati differential equation (3.5) and $\Phi(t, s)$ is given by (3.7).

Correspondingly, we consider the sequence of control problems of minimizing

$$
J_{s}^{N}(u)=\left\langle x^{N}(T), \mathscr{S}_{x}^{N}(T)\right\rangle+\int_{s}^{T}\left[\left\|\mathscr{C}_{x}^{N}(t)\right\|^{2}+u(t)^{T} \mathscr{R} u(t)\right] d t,
$$

where $x^{N}(t)=x^{N}\left(t ; s, p^{N} x_{0}, u\right)$ is the unique solution of (4.5). The optimal control is given by the feedback law

$$
\begin{aligned}
\bar{u}_{s}^{N}(t) & =-\mathscr{R}^{-1}\left(\mathscr{B}^{N}\right)^{*} \mathscr{P}^{N}(t) \Phi^{N}(t, s) p^{N} x_{0} \\
& =-\mathscr{R}^{-1}\left(\mathscr{B}^{N}\right)^{*} \mathscr{P}^{N}(t) \Phi^{N}(t, s) x_{0}, \quad s \leqq t \leqq T,
\end{aligned}
$$

where the strongly continuous, positive semidefinite operator $\mathscr{P}^{N}(t): \mathscr{X} \rightarrow \mathscr{X}$ and the strongly continuous evolution operator $\Phi^{N}(t, s): \mathscr{X} \rightarrow \mathscr{X}$ are defined by the equations

$$
\begin{aligned}
\mathscr{P}^{N}(t)_{x}=\mathscr{S}^{N}(T & -t)^{*} p^{N} \mathscr{G}_{p^{N}} \Phi^{N}(T, t)_{x} \\
& +\int_{t}^{T} \mathscr{S}^{N}(\tau-t)^{*} p^{N} \mathscr{C}^{*} \mathscr{C}^{N} \Phi^{N}(\tau, t) x d t, \quad t \leqq T,
\end{aligned}
$$

and

$$
\Phi^{N}(t, s) x=\mathscr{S}^{N}(t-s) x-\int_{s}^{t} \mathscr{S}^{N}(t-\tau) \mathscr{B}^{N} \mathscr{R}^{-1}\left(\mathscr{B}^{N}\right)^{*} \mathscr{P}^{N}(\tau) \Phi^{N}(\tau, s) x d \tau,
$$

for $x \in \mathscr{X}$. It follows immediately from (4.11) and the fact that $\mathscr{P}^{N}(t)$ is selfadjoint that

$$
\mathscr{P}^{N}(t)=p^{N} \mathscr{P}^{N}(t) p^{N}, \quad t \leqq T .
$$


This in turn implies, by (4.12), that

$$
p^{N} \Phi^{N}(t, s)=\Phi^{N}(t, s) p^{N}, \quad s \leqq t \leqq T .
$$

Note that these two facts justify the second equation in (4.12). Moreover, the optimal cost of (4.9), (4.5) is given by

$$
J_{s}^{N}\left(\bar{u}_{s}^{N}\right)=\left\langle x_{0}, \mathscr{P}^{N}(s) x_{0}\right\rangle .
$$

We remark that $\mathscr{P}^{N}(t)$, regarded as an operator on $\mathscr{X}^{N}$, satisfies the following finite dimensional Riccati differential equation

$$
\begin{aligned}
& \frac{d}{d t} \mathscr{P}^{N}(t)+\left(\mathscr{A}^{N}\right)^{*} \mathscr{P}^{N}(t)+\mathscr{P}^{N}(t) \mathscr{A}^{N} \\
& -\mathscr{P}^{N}(t) \mathscr{B}^{N} \mathscr{R}^{-1}\left(\mathscr{B}^{N}\right)^{*} \mathscr{P}^{N}(t)+p^{N} \mathscr{C}^{*} \mathscr{C} p^{N}=0, \quad t \leqq T, \\
& \mathscr{P}^{N}(t)=p^{N} \mathscr{G} p^{N} .
\end{aligned}
$$

Obviously, the most interesting question is how the original system (4.3) behaves when the optimal feedback control (4.8) is replaced by the approximate control law

$$
\hat{u}_{s}^{N}(t)=-\mathscr{R}^{-1}\left(\mathscr{B}^{N}\right) * \mathscr{P}^{N}(t) \hat{\Phi}^{N}(t, s) x_{0},
$$

where $\hat{\Phi}^{N}(t, s)$ denotes the corresponding closed loop evolution operator on $\mathscr{X}$ which is defined by

$$
\hat{\Phi}^{N}(t, s) x=\mathscr{S}(t-s) x-\int_{s}^{t} \mathscr{S}(t-\tau) \mathscr{B} \mathscr{R}^{-1}\left(\mathscr{B}^{N}\right)^{*} \mathscr{P}^{N}(\tau) \hat{\Phi}^{N}(\tau, s) x d \tau
$$

$$
\text { for } x \in \mathscr{X} \text { and } s \leqq t \leqq T \text {. }
$$

All the desired convergence results are contained in the next theorem which is a straight forward consequence of Theorems 6.1-6.3 in [20]. For the convenience of the reader we present the main ideas of the proof.

THEOREM 4.3. Let us assume that

(i) There exist constants $M \geqq 1, \omega \in \mathbb{R}$ such that

$$
\left\|\mathscr{S}^{N}(t)\right\| \leqq M e^{\omega t}, \quad t \geqq 0, \quad N=1,2, \cdots ;
$$

(ii) For every $x \in \mathscr{X}$

$$
\lim _{N \rightarrow \infty} \mathscr{S}^{N}(t) p^{N} x=\mathscr{Y}(t) x, \quad \lim _{N \rightarrow \infty} \mathscr{S}^{N}(t)^{*} p^{N} x=\mathscr{S}(t)^{*} x
$$

uniformly on $[0, T]$;

(iii) $\lim _{N \rightarrow \infty} \mathscr{B}^{N} \xi=\mathscr{B} \xi$ for every $\xi \in \mathbb{R}^{l}$.

Then, for every $x_{0} \in \mathscr{X}$,

(a) $\lim _{N \rightarrow \infty} J_{s}^{N}\left(\bar{u}_{s}^{N}\right)=\lim _{N \rightarrow \infty} J_{s}\left(\hat{u}_{s}^{N}\right)=J_{s}\left(\bar{u}_{s}\right)$,

(b) $\lim _{N \rightarrow \infty} \bar{u}_{s}^{N}(t)=\lim _{N \rightarrow \infty} \hat{u}_{s}^{N}(t)=\bar{u}_{s}(t)$,

(c) $\lim _{N \rightarrow \infty} \Phi^{N}(t, s) x_{0}=\lim _{N \rightarrow \infty} \hat{\Phi}^{N}(t, s) x_{0}=\Phi(t, s) x_{0}$,

(d) $\lim _{N \rightarrow \infty} \mathscr{P}^{N}(s) x_{0}=\mathscr{P}(s) x_{0}$

and the limits are uniform on the domain $0 \leqq s \leqq t \leqq T$. If range $\mathscr{G}$ is finite dimensional, then $\mathscr{P}^{N}(s)$ converes to $\mathscr{P}(s)$ in the uniform operator topology, uniformly on the interval $[0, T]$. 
Proof. Let us introduce the operators $\mathscr{F}_{s}(t): L^{2}\left(s, T ; \mathbb{R}^{l}\right) \rightarrow \mathscr{X}, \mathscr{G}_{s}: \mathscr{X} \rightarrow L^{2}\left(s, T ; \mathbb{R}^{l}\right)$, $\mathscr{R}_{s}: L^{2}\left(s, T ; \mathbb{R}^{l}\right) \rightarrow L^{2}\left(s, T ; \mathbb{R}^{l}\right)$ by defining

$$
\begin{aligned}
& \mathscr{F}_{s}(t) u=\int_{s}^{t} \mathscr{S}(t-\tau) \mathscr{B} u(\tau) d \tau, \\
& \mathscr{G}_{s} x=\mathscr{F}_{s}(T)^{*} \mathscr{G} \mathscr{S}(T-s) x+\int_{s}^{T} \mathscr{F}_{s}(\tau)^{*} \mathscr{C} * \mathscr{C} \mathscr{S}(\tau-s) x d \tau, \\
& \mathscr{R}_{s} u=\mathscr{F}_{s}(T)^{*} \mathscr{G} \mathscr{F}_{s}(T) u+\int_{s}^{T} \mathscr{F}_{s}(\tau)^{*} \mathscr{C}^{*} \mathscr{C F}_{s}(\tau) u d \tau+\mathscr{R} u
\end{aligned}
$$

for $u \in L^{2}\left(s, T ; \mathbb{R}^{l}\right)$ and $x \in \mathscr{X}$. Of course, $\mathscr{R} u$ is defined by $(\mathscr{R} u)(t)=\mathscr{R} u(t), s \leqq t \leqq T$. Then it is easy to see that the Fréchet derivative of $J_{s}$ with respect to $u$ is given by $J_{s}^{\prime}(u)=2 \mathscr{R}_{s} u+2 \mathscr{G}_{s} x_{0}$. Since the optimal control $\bar{u}_{s}$ satisfies $J_{s}^{\prime}\left(\bar{u}_{s}\right)=0$, this implies

$$
\bar{u}_{s}=-\mathscr{R}_{s}^{-1} \mathscr{G}_{s} x_{0} .
$$

Analogously, we get

$$
\bar{u}_{s}^{N}=-\left(\mathscr{R}_{s}^{N}\right)^{-1} \mathscr{G}_{s}^{N} p^{N} x_{0}=-\left(\mathscr{R}_{s}^{N}\right)^{-1} \mathscr{G}_{s}^{N} x_{0}
$$

where $\mathscr{R}_{s}^{N}, \mathscr{G}_{s}^{N}, \mathscr{F}_{s}^{N}$ are defined as above with $\mathscr{S}(t), \mathscr{B}, \mathscr{C}, \mathscr{G}$ replaced by $\mathscr{S}^{N}(t), \mathscr{B}^{N}$, $\mathscr{C} p^{N}, p^{N} \mathscr{G} p^{N}$, respectively. Combining these formulae with (3.7), (4.8) and (4.12), (4.10), we get

$$
\begin{aligned}
& \Phi(t, s) x_{0}=\mathscr{S}(t-s) x_{0}-\mathscr{F}_{s}(t) \mathscr{R}_{s}^{-1} \mathscr{G}_{s} x_{0}, \\
& \Phi^{N}(t, s) x_{0}=\mathscr{S}^{N}(t-s) x_{0}-\mathscr{F}_{s}^{N}(t)\left(\mathscr{R}_{s}^{N}\right)^{-1} \mathscr{G}_{s}^{N} x_{0}
\end{aligned}
$$

for every $s \in[0, T]$ and every $t \in[s, T]$.

We have shown in Theorem 4.2 that $\mathscr{F}_{s}^{N}(t)$ converges to $\mathscr{F}_{s}(t)$ in the uniform operator topology, uniformly for $0 \leqq s \leqq t \leqq T$. This implies that for every $x \in \mathscr{X}$

$$
\lim _{N \rightarrow \infty} \mathscr{G}_{s}^{N} x=\mathscr{G}_{s} x
$$

uniformly on $[0, T]$ and moreover $\left\|\mathscr{R}_{s}^{N}-\mathscr{R}_{s}\right\| \rightarrow 0$, also uniformly on $[0, T]$. Choosing $\varepsilon>0$ such that $\xi^{T} \mathscr{R} \xi \geqq \varepsilon|\xi|^{2}$ for $\xi \in \mathbb{R}^{l}$, we obtain

and hence

$$
\left\|\mathscr{R}_{s}^{N} u\right\| \geqq \varepsilon\|u\|, \quad u \in L^{2}\left(s, T ; \mathbb{R}^{l}\right), \quad N=1,2, \cdots
$$

$$
\lim _{N \rightarrow \infty}\left\|\left(\mathscr{R}_{s}^{N}\right)^{-1}-\mathscr{R}_{s}^{-1}\right\|=0
$$

uniformly on $[0, T]$.

It follows immediately from (4.22)-(4.25) that $\Phi^{N}(t, s)$ converges strongly to $\Phi(t, s)$. By (4.11) and (3.6), this implies the strong convergence of the Riccati operators $\mathscr{P}^{N}(s)$ to $\mathscr{P}(s)$. Now the convergence result on $\hat{\Phi}^{N}(t, s)$ follows from the inequality

$$
\begin{aligned}
\| \Phi(t, s) x- & \hat{\Phi}^{N}(t, s) x \| \\
\leqq & \int_{s}^{t}\left\|\mathscr{S}(t-\tau) \mathscr{B} \mathscr{R}^{-1}\right\|\left\|\left[\left(\mathscr{B}^{N}\right)^{*} \mathscr{P}^{N}(\tau)-\mathscr{B}^{*} \mathscr{P}(\tau)\right] \Phi(\tau, s) x\right\| d \tau \\
& \quad+\int_{s}^{t}\left\|\mathscr{S}(t-\tau) \mathscr{B}^{-1}\left(\mathscr{B}^{N}\right)^{*} \mathscr{P}^{N}(\tau)\right\|\left\|\hat{\Phi}^{N}(\tau, s) x-\Phi(\tau, s) x\right\| d \tau
\end{aligned}
$$


and Gronwall's lemma. Thus we have established the statements (c) and (d). Statement (b) follows from (c) and (d), since the control functions $\bar{u}_{s}, \bar{u}_{s}^{N}, \hat{u}_{s}^{N}$ are given by (4.10), (4.12), (4.19) respectively. Statement (a) is an immediate consequence of (b) and (d), since $J_{s}^{N}\left(\bar{u}_{s}^{N}\right)=\left\langle x_{0}, \mathscr{P}^{N}(s) x_{0}\right\rangle$ and $J_{s}\left(\bar{u}_{s}\right)=\left\langle x_{0}, \mathscr{P}(s) x_{0}\right\rangle$. If range $\mathscr{G}$ is finite dimensional, then the convergence of $\mathscr{P}^{N}(s)$ in the uniform operator topology can be established by analogous considerations as those in the proof of Theorem 4.2 (see [4]), again by the use of (4.13) and (3.6).

5. A special approximation scheme.

5.1. General ideas. In this section we present a general idea how to construct special approximation schemes satisfying the assumptions of $\S 4$.

Let the sequence $X^{N}, N=1,2, \cdots$, of subspaces of $M^{2}$ be defined by

$$
X^{N}=\left\{\phi \in M^{2} \mid \phi=\hat{e}_{0}^{N} \alpha_{0}+\sum_{i=1}^{p} \sum_{j=1}^{k_{N}} \hat{e}_{i j}^{N} \alpha_{i j}, \alpha_{0}, \alpha_{i j} \in \mathbb{R}^{n}\right\},
$$

where the "basis elements" $\hat{e}_{0}^{N}, \hat{e}_{i j}^{N}$ are given by

$$
\hat{e}_{0}^{N}=(I, 0), \quad \hat{e}_{i j}^{N}=\left(0, e_{i j}^{N} I\right),
$$

i.e., $X^{N}=\mathbb{R}^{n} \times Y^{N}$ with $Y^{N}=\operatorname{span}\left(e_{11}^{N} I, \cdots, e_{p k_{N}}^{N} I\right) \subset L^{2}\left(-h, 0 ; \mathbb{R}^{n}\right)$. It is clear that $\operatorname{dim} X^{N}=\left(p k_{N}+1\right) n$. We assume that

$$
\begin{aligned}
& e_{i j}^{N} \mid\left[-h_{i},-h_{i-1}\right) \in W^{1,2}\left(-h_{i},-h_{i-1} ; \mathbb{R}\right), \\
& e_{i j}^{N}(\tau)=0 \text { for } \tau \notin\left[-h_{i},-h_{i-1}\right) .
\end{aligned}
$$

Without restriction we may further assume that $e_{i j}^{N}$ is right-hand continuous on $[-h, 0)$.

Because of the product space structure of the subspaces $X^{N}$ the orthogonal projections $p^{N}: M^{2} \rightarrow X^{N}$ are given by

$$
p^{N} \phi=\left(\phi^{0}, \pi^{N} \phi^{1}\right) \text { for } \phi=\left(\phi^{0}, \phi^{1}\right) \in M^{2},
$$

where $\pi^{N}$ is the orthogonal projection $L^{2}\left(-h, 0 ; \mathbb{R}^{n}\right) \rightarrow Y^{N}$. We introduce

$$
\hat{E}^{N}=\left(\hat{e}_{0}^{N}, \hat{e}_{11}^{N}, \cdots, \hat{e}_{p k_{N}}^{N}\right)
$$

and denote by $\alpha^{N}(\phi)=\operatorname{col}\left(\alpha_{0}^{N}, \alpha_{11}^{N}, \cdots, \alpha_{p k_{N}}^{N}\right) \in \mathbb{R}^{n\left(p k_{N}+1\right)}$ the coordinate vector of an element $\phi \in X^{N}$, i.e.,

$$
\phi=\hat{E}^{N} \alpha^{N}(\phi), \quad \phi \in X^{N}
$$

An easy calculation shows

$$
\alpha^{N}\left(p^{N} \phi\right)=\left(Q^{N}\right)^{-1} d^{N}(\phi), \quad \phi \in M^{2},
$$

where

$$
\begin{aligned}
& d^{N}(\phi)=\left\langle\hat{E}^{N}, \phi\right\rangle_{M^{2}}=\operatorname{col}\left(\phi^{0},\left\langle e_{11}^{N}, \phi^{1}\right\rangle_{L^{2}}, \cdots,\left\langle e_{p k_{N}}^{N}, \phi^{1}\right\rangle_{L^{2}}\right), \\
& Q^{N}=\left\langle\hat{E}^{N}, \hat{E}^{N}\right\rangle_{M^{2}}=\operatorname{diag}\left(I, q_{1}^{N} \otimes I, \cdots, q_{p}^{N} \otimes I\right),
\end{aligned}
$$

where $q_{i}^{N}=\left(\left\langle e_{i j}^{N}, e_{i k}^{N}\right\rangle_{L^{2}}\right)_{j, k=1, \cdots, k_{N}}$ and $I$ is the $n \times n$ identity matrix. For elements in $X^{N}$ the inner product has the representation

$$
\langle\phi, \psi\rangle_{M^{2}}=\alpha^{N}(\phi)^{T} Q^{N} \alpha^{N}(\psi), \quad \phi, \psi \in X^{N} .
$$


Since for elements $\phi=\left(\phi^{0}, \phi^{1}\right) \in X^{N}$ in general $\phi^{0} \neq \lim _{t \uparrow 0} \phi^{1}(\tau)$ and $\phi^{1}$ may have jumps of arbitrary size at the delay points $-h_{i}$, it is clear that $X^{N}$ is not contained in dom $A$ nor in dom $A^{*}$. However, the operators $A$ and $A^{*}$ can formally be extended to all of $X^{N}$ in the following way:

$$
\begin{gathered}
{[A \phi]^{0}=A_{0} \phi^{0}+\sum_{i=1}^{p} A_{i} \phi^{1}\left(-h_{i}\right)+\int_{-h}^{0} A_{01}(\tau) \phi^{1}(\tau) d \tau} \\
{[A \phi]^{1}(\tau)=\frac{d^{+}}{d \theta} \phi^{1}(\tau)+\delta_{0}(\tau)\left(\phi^{0}-\lim _{\tau \uparrow 0} \phi^{1}(\tau)\right)} \\
\quad+\sum_{i=1}^{p-1} \delta_{i}(\tau)\left(\phi^{1}\left(-h_{i}\right)-\lim _{\tau \uparrow-h_{i}} \phi^{1}(\tau)\right), \\
{\left[A^{*} \psi\right]^{0}=\lim _{\tau \uparrow 0} \psi^{1}(\tau)+A_{0}^{T} \psi^{0},} \\
{\left[A^{*} \psi\right]^{1}(\tau)=A_{01}^{T}(\tau) \psi^{0}-\frac{d^{+}}{d \theta} \psi^{1}(\tau)} \\
\quad+\sum_{i=1}^{p-1} \delta_{i}(\tau)\left(A_{i}^{T} \psi^{0}-\psi^{1}\left(-h_{i}\right)+\lim _{\tau \uparrow-h_{i}} \psi^{1}(\tau)\right) \\
+\delta_{p}(\tau)\left(A_{p}^{T} \psi^{0}-\psi^{1}(-h)\right)
\end{gathered}
$$

for $\phi, \psi \in X^{N}$, where $\delta_{i}$ denotes the Dirac delta impulse at $-h_{i}, i=0, \cdots, p$. Below we shall introduce the operators $A^{N}$ and $\left(A^{N}\right)^{*}$ by projecting these formal extensions formally back into the subspace $X^{N}$. Since jumps of the function components of elements in $X^{N}$ may occur at $\tau=-h_{1}$, we have two possible interpretations of $\delta_{i}$ as a functional on $X^{N}$, namely the evaluation of either the right-hand or the left-hand limit at $-h_{i}$. Correspondingly we introduce the following two types of approximate delta impulses which can be obtained. We define

$$
\begin{aligned}
\delta_{i,+}^{N} & =\hat{E}^{N} \gamma_{i,+}^{N}, & i & =1, \cdots, p, \\
\delta_{i,-}^{N} & =\hat{E}^{N} \gamma_{i,-}^{N}, & i & =0, \cdots, p-1,
\end{aligned}
$$

where

$$
\begin{aligned}
& Q^{N} \gamma_{i,+}^{N}=\operatorname{col}\left(0, e_{11}^{N}\left(-h_{i}\right), \cdots, e_{p k_{N}}^{N}\left(-h_{i}\right)\right), \\
& Q^{N} \gamma_{i,-}^{N}=\operatorname{col}\left(0, \lim _{\tau \uparrow-h_{i}} e_{11}^{N}(\tau), \cdots, \lim _{\tau \uparrow-h_{i}} e_{p k_{N}}^{N}(\tau)\right) .
\end{aligned}
$$

The following lemma describes the action of the approximating delta-impulses.

LEMMA 5.1. For any $x \in \mathbb{R}^{n}$ and $\phi \in M^{2}$

$$
\begin{aligned}
& \left\langle\delta_{i,+}^{N} x, \phi\right\rangle_{M^{2}}=x^{T}\left(\pi^{N} \phi^{1}\right)\left(-h_{i}\right), \quad i=1, \cdots, p, \\
& \left\langle\delta_{i,-}^{N} x, \phi\right\rangle_{M^{2}}=x^{T} \lim _{\tau \uparrow-h_{i}}\left(\pi^{N} \phi^{1}\right)(\tau), \quad i=0, \cdots, p-1 .
\end{aligned}
$$

Proof. Using (5.2), (5.3) and the definition of $\delta_{i,+}^{N}$, we get

$$
\begin{aligned}
\left\langle\delta_{i,+}^{N} x, \phi\right\rangle_{M^{2}} & =\left\langle\hat{E}^{N} \gamma_{i,+}^{N} x, p^{N} \phi\right\rangle_{M^{2}}=\left(\gamma_{i,+}^{N} x\right)^{T} Q^{N} \alpha\left(p^{N} \phi\right) \\
& =x^{T}\left(0, e_{11}^{N}\left(-h_{i}\right), \cdots, e_{p k_{N}}^{N}\left(-h_{i}\right)\right) \alpha^{N}\left(p^{N} \phi\right) \\
& =x^{T}\left(\pi^{N} \phi^{1}\right)\left(-h_{i}\right) .
\end{aligned}
$$

The proof for $\delta_{i,-}^{N}$ is analogous. 
The following definition of the operators $A^{N}$ is obtained by formally projecting $A \phi$ as given by (5.4), (5.5) into $X^{N}$ and putting $p^{N} \delta_{i}=\delta_{i,-}^{N}, i=0, \cdots, p-1$.

Definition 5.2. For any $\phi=\left(\phi^{0}, \phi^{1}\right) \in X^{N}$ we define

$$
\begin{array}{r}
A^{N} \phi=\left(A_{0} \phi^{0}+\sum_{i=1}^{p} A_{i} \phi^{1}\left(-h_{i}\right)+\int_{-h}^{0} A_{01}(\tau) \phi^{1}(\tau) d \tau, \pi^{N}\left(\frac{d^{+}}{d \theta} \phi^{1}\right)\right) \\
+\delta_{0,-}^{N}\left(\phi^{0}-\lim _{\tau \uparrow 0} \phi^{1}(\tau)\right)+\sum_{i=1}^{p-1} \delta_{i,-}^{N}\left(\phi^{1}\left(-h_{i}\right)-\lim _{\tau \uparrow-h_{i}} \phi^{1}(\tau)\right) .
\end{array}
$$

The adjoint operators $\left(A^{N}\right)^{*}$ are given in

LEMMA 5.3. For any $\psi=\left(\psi^{0}, \psi^{1}\right) \in X^{N}$ the operator $\left(A^{N}\right)^{*}$ is given by

$$
\begin{aligned}
\left(A^{N}\right)^{*} \psi= & \left(\lim _{\tau \uparrow 0} \psi^{1}(\tau)+A_{0}^{T} \psi^{0}, \pi^{N}\left(A_{01}^{T} \psi^{0}-\frac{d^{+}}{d \theta} \psi^{1}\right)\right) \\
& +\sum_{i=1}^{p-1} \delta_{i,+}^{N}\left(A_{i}^{T} \psi^{0}+\lim _{\tau \uparrow-h_{i}} \psi^{1}(\tau)-\psi^{1}\left(-h_{i}\right)\right) \\
& +\delta_{p,+}^{N}\left(A_{p}^{T} \psi^{0}-\psi^{1}(-h)\right) .
\end{aligned}
$$

Proof. By definition of the adjoint operator we get

$$
\begin{aligned}
&\left\langle\left(A^{N}\right)^{*} \psi, \phi\right\rangle_{M^{2}}=\langle\psi\left.A^{N} \phi\right\rangle_{M^{2}} \\
&=\left(\psi^{0}\right)^{T}\left[A_{0} \phi^{0}+\sum_{i=1}^{p} A_{i} \phi^{1}\left(-h_{i}\right)+\int_{-h}^{0} A_{01}(\tau) \phi^{1}(\tau) d \tau\right] \\
&+\left\langle\psi^{1}, \pi^{N}\left(\frac{d^{+}}{d \theta} \phi^{1}\right)\right\rangle_{L^{2}}+\left\langle\psi, \delta_{0,-}^{N}\left(\phi^{0}-\lim _{\tau \uparrow 0} \psi^{1}(\tau)\right)\right\rangle_{M^{2}} \\
&+\sum_{i=1}^{p-1}\left\langle\psi, \delta_{i,-}^{N}\left(\phi^{1}\left(-h_{i}\right)-\lim _{\tau \uparrow-h_{i}} \phi^{1}(\tau)\right)\right\rangle_{M^{2}}
\end{aligned}
$$

for any $\phi=\left(\phi^{0}, \phi^{1}\right), \psi=\left(\psi^{0}, \psi^{1}\right)$ in $X^{N}$. By Lemma 5.1 we see

$$
\begin{aligned}
& \left\langle\psi, \delta_{0,-}^{N}\left(\phi^{0}-\lim _{\tau \uparrow 0} \phi^{1}(\tau)\right)\right\rangle_{M^{2}}=\lim _{\tau \uparrow 0} \psi^{1}(\tau)^{T}\left(\phi^{0}-\lim _{\tau \uparrow 0} \phi^{1}(\tau)\right), \\
& \left\langle\psi, \delta_{i,-}^{N}\left(\phi^{1}\left(-h_{i}\right)-\lim _{\tau \uparrow-h_{i}} \phi^{1}(\tau)\right)\right\rangle_{M^{2}} \\
& \quad=\lim _{\tau \uparrow-h_{i}} \psi^{1}(\tau)^{T}\left(\phi^{1}\left(-h_{i}\right)-\lim _{\tau \uparrow-h_{i}} \phi^{1}(\tau)\right), \quad i=1, \cdots, p-1 .
\end{aligned}
$$

Furthermore,

$$
\begin{aligned}
\left\langle\psi^{1}, \pi^{N}\left(\frac{d^{+}}{d \theta} \phi^{1}\right)\right\rangle_{L^{2}}= & \left\langle\psi^{1}, \frac{d^{+}}{d \theta} \phi^{1}\right\rangle_{L^{2}}=\sum_{i=1}^{p} \int_{-h_{i}}^{-h_{i-1}} \psi^{1}(\tau)^{T}\left(\frac{d^{+}}{d \theta} \phi^{1}\right)(\tau) d \tau \\
= & \sum_{i=1}^{p}\left[\lim _{\tau \uparrow-h_{i-1}} \psi^{1}(\tau)^{T} \phi^{1}(\tau)-\psi^{1}\left(-h_{i}\right)^{T} \phi^{1}\left(-h_{i}\right)\right] \\
& -\left\langle\pi^{N}\left(\frac{d^{+}}{d \theta} \psi^{1}\right), \phi^{1}\right\rangle_{L^{2}}
\end{aligned}
$$

and

$$
\left(\psi^{0}\right)^{T} \int_{-h}^{0} A_{01}(\tau) \phi^{1}(\tau) d \tau=\left\langle A_{01}^{T} \psi^{0}, \phi^{1}\right\rangle_{L^{2}}=\left\langle\pi^{N}\left(A_{01}^{T} \psi^{0}\right), \phi^{1}\right\rangle_{L^{2}}
$$


Altogether we have

$$
\begin{aligned}
\left\langle\left(A^{N}\right)^{*} \psi, \phi\right\rangle_{M^{2}=\left[\lim _{\tau \uparrow 0}\right.} & \left.\psi^{1}(\tau)+A_{0}^{T} \psi^{0}\right]^{T} \phi^{0} \\
& +\sum_{i=1}^{p-1}\left[A_{i}^{T} \psi^{0}+\lim _{\tau \uparrow-h_{i}} \psi^{1}(\tau)-\psi^{1}\left(-h_{i}\right)\right]^{T} \phi^{1}\left(-h_{i}\right) \\
& +\left[A_{p}^{T} \psi^{0}-\psi^{1}(-h)\right]^{T} \phi^{1}(-h)+\left\langle\pi^{N}\left(A_{01}^{T} \psi^{0}-\frac{d^{+}}{d \theta} \psi^{1}\right), \phi^{1}\right\rangle_{L^{2}}
\end{aligned}
$$

The result now follows using Lemma 5.1.

Note, that $\left(A^{N}\right)^{*} \psi$ can formally be obtained by projecting $A^{*} \psi$ as given by (5.6), (5.7) into $X^{N}$ but now putting $p^{N} \delta_{i}=\delta_{i,+}^{N}, i=1, \cdots, p$. Without any additional assumption we have the following.

LEMMA 5.4. Hypothesis $(\mathrm{H} 2)$ is valid for the sequence $A^{N}, N=1,2, \cdots$ and therefore also for the sequence $\left(A^{N}\right)^{*}, N=1,2, \cdots$.

Proof. We introduce an equivalent inner product on $M^{2}$ by

$$
\langle\phi, \psi\rangle_{g}=\left(\phi^{0}\right)^{T} \psi^{0}+\int_{-h}^{0} \phi^{1}(\tau)^{T} \psi^{1}(\tau) g(\tau) d \tau, \quad \phi, \psi \in M^{2}
$$

where the weighting function $g$ is right-hand continuous on $[-h, 0)$ and

$$
g(\tau)=p-i+1 \quad \text { for } \tau \in\left[-h_{i},-h_{i-1}\right), \quad i=1, \cdots, p .
$$

It is clear that the corresponding norm $\|\cdot\|_{g}$ on $M^{2}$ is equivalent to the original norm,

$$
\|\phi\| \leqq\|\phi\|_{g} \leqq \sqrt{p}\|\phi\|, \quad \phi \in M^{2} .
$$

Since $\left(\phi^{0}, \phi^{1} g\right) \in X^{N}$ for any $\phi \in X^{N}$, we obtain from Lemma 5.1

$$
\left\langle\delta_{i,-}^{N} x, \phi\right\rangle_{g}=(p-i) x^{T} \lim _{\tau \uparrow-h_{i}} \phi^{1}(\tau), \quad i=0, \cdots, p-1,
$$

for $x \in \mathbb{R}^{n}$ and $\phi \in X^{N}$. Using this equation and Definition 5.2, we get for $\phi \in X^{N}$

$$
\begin{aligned}
\left\langle A^{N} \phi, \phi\right\rangle_{g}=\left[A_{0} \phi^{0}+\sum_{i=1}^{p} A_{i} \phi^{i}\left(-h_{i}\right)+\int_{-h}^{0} A_{01}(\tau) \phi^{1}(\tau) d \tau\right]^{T} \phi^{0} \\
+\left\langle\pi^{N}\left(\frac{d^{+}}{d \theta} \phi^{1}\right), \phi^{1} g\right\rangle_{L^{2}}+p\left[\phi^{0}-\lim _{\tau \uparrow 0} \phi^{1}(\tau)\right]^{T} \lim _{\tau \uparrow 0} \phi^{1}(\tau) \\
+\sum_{i=1}^{p-1}(p-i)\left[\phi^{1}\left(-h_{i}\right)-\lim _{\tau \uparrow-h_{i}} \phi^{1}(\tau)\right]^{T} \lim _{\tau \uparrow-h_{i}} \phi^{1}(\tau) .
\end{aligned}
$$

Obviously $\pi^{N}\left(\phi^{1} g\right)=\phi^{1} g$ and hence

$$
\begin{aligned}
\left\langle\pi^{N}\left(\frac{d^{+}}{d \theta} \phi^{1}\right), \phi^{1} g\right\rangle_{L^{2}} & =\left\langle\frac{d^{+}}{d \theta} \phi^{1}, \phi^{1} g\right\rangle_{L^{2}} \\
& =\sum_{i=1}^{p}(p-i+1) \int_{-h_{i}}^{-h_{i-1}} \dot{\phi}^{1}(\tau)^{T} \phi^{1}(\tau) d \tau \\
& =\frac{1}{2} \sum_{i=1}^{p}(p-i+1)\left[\lim _{\tau \uparrow-h_{i-1}}\left|\phi^{1}(\tau)\right|^{2}-\left|\phi^{1}\left(-h_{i}\right)\right|^{2}\right] .
\end{aligned}
$$


Using this and several times the inequality $\alpha \beta \leqq \frac{1}{2} \alpha^{2}+\frac{1}{2} \beta^{2}$, we get for $\phi \in X^{N}$

$$
\begin{aligned}
& \left\langle A^{N} \phi, \phi\right\rangle_{g} \leqq\left(\left|A_{0}\right|+\frac{1}{2} \sum_{i=1}^{p}\left|A_{i}\right|^{2}+\left\|A_{01}\right\|_{L^{2}}\right)\|\phi\|_{g}^{2} \\
& +\frac{1}{2} \sum_{i=1}^{p}\left|\phi^{1}\left(-h_{i}\right)\right|^{2}+\frac{p}{2}\left|\phi^{0}\right|^{2}-\frac{p}{2} \lim _{\tau \uparrow 0}\left|\phi^{1}(\tau)\right|^{2} \\
& \left.+\frac{1}{2} \sum_{i=1}^{p}(p-i+1) \lim _{\tau \uparrow-h_{i-1}}\left|\phi^{1}(\tau)\right|^{2}-\left|\phi^{1}\left(-h_{i}\right)\right|^{2}\right] \\
& +\frac{1}{2} \sum_{i=2}^{p}(p-i+1)\left[\left|\phi^{1}\left(-h_{i-1}\right)\right|^{2}-\lim _{\tau \uparrow-h_{i-1}}\left|\phi^{1}(\tau)\right|^{2}\right] \\
& \leqq \omega\|\phi\|_{g}^{2}
\end{aligned}
$$

with $\omega=p / 2+\left|A_{0}\right|+\frac{1}{2} \sum_{i=1}^{p}\left|A_{i}\right|^{2}+\left\|A_{01}\right\|_{L^{2}}$. This proves $\left(\mathrm{H} 2^{*}\right)$ with $\|\cdot\|_{N}=\|\cdot\|_{g}$ for all $N$. Since $\left\|S^{N}(t)\right\|=\left\|S^{N}(t)^{*}\right\|$ the proof is finished.

In order to verify hypothesis (H3), we need additional assumptions concerning the convergence properties of $\pi^{N} \phi^{1}, N=1,2, \cdots$, for $\phi$ in a suitably restricted subset of $M^{2}$. Observe, that we get from (5.1)

$$
\pi^{N} \phi^{1}=\sum_{j=1}^{p} \pi_{j}^{N}\left(\phi^{1} \mid\left[-h_{j},-h_{j-1}\right)\right),
$$

where $\pi_{j}^{N}$ is the orthogonal projection $L^{2}\left(-h_{j},-h_{j-1} ; \mathbb{R}^{n}\right) \rightarrow \operatorname{span}\left(e_{j 1}^{N} I, \cdots, e_{j k_{N}}^{N} I\right)$. We define the sets $\tilde{D}$ and $\tilde{D}^{*}$ by

$$
\begin{aligned}
& \tilde{D}=\left\{\phi \in M^{2} \mid \phi^{0}=\phi^{1}(0), \phi^{1} \in W^{2,2}\left(-h, 0 ; \mathbb{R}^{n}\right)\right\}, \\
& \tilde{D}^{*}=\left\{\psi \in M^{2} \mid \psi^{1}(-h)=A_{p}^{T} \psi^{0}, \psi^{1}+\sum_{j=1}^{p-1} A_{i}^{T} \psi^{0} \chi_{\left[-h_{i},-h_{i-1}\right.} \in W^{1,2}\left(-h, 0 ; \mathbb{R}^{n}\right),\right. \\
& \left.\quad \text { and } A_{01}^{T} \psi^{0}-\frac{d^{+}}{d \theta} \psi^{1} \in W^{1,2}\left(-h_{i},-h_{i-1} ; \mathbb{R}^{n}\right), i=1, \cdots, p\right\} .
\end{aligned}
$$

Obviously, dom $A^{2} \subset \tilde{D} \subset \operatorname{dom} A$ and dom $\left(A^{*}\right)^{2} \subset \tilde{D}^{*} \subset \operatorname{dom} A^{*}$. Furthermore we put

$$
\kappa(N)=\max \left(\left\|\delta_{0,-}^{N}\right\|, \cdots,\left\|\delta_{p-1,-}^{N}\right\|,\left\|\delta_{1,+}^{N}\right\|, \cdots,\left\|\delta_{p,+}^{N}\right\|\right),
$$

where $\left\|\delta_{i, \pm}^{N}\right\|$ is the norm of $\delta_{i, \pm}^{N}$ considered as an operator $\mathbb{R}^{n} \rightarrow M^{2}$. We impose the following hypothesis:

(A) There exists a sequence $\rho(N)$ with $\lim _{N \rightarrow \infty} \rho(N)=0$ such that for $i=1, \cdots, p$

(i) $(1+\kappa(N))\left\|f-\pi_{i}^{N} f\right\|_{L^{\infty}}+\left\|f-\pi_{i}^{N} f\right\|_{L^{2}}$

$$
+\left\|\frac{d}{d \theta}\left(f-\pi_{i}^{N} f\right)\right\|_{L^{2}} \leqq \rho(N)\|f\|_{W^{2,2}}
$$

for all $f \in W^{2,2}\left(-h_{i},-h_{i-1} ; \mathbb{R}^{n}\right)$, and

(ii) $\left\|f-\pi_{i}^{N} f\right\|_{L^{2}} \leqq \rho(N)\|f\|_{W^{1,2}}$

for all $f \in W^{1,2}\left(-h_{i},-h_{i-1} ; \mathbb{R}^{n}\right)$.

Lemma 5.5. Assume that (A) is satisfied. Then the following is true:

(a) Assumption (H1) is satisfied.

(b) For any $\phi \in \tilde{D}$

$$
\left\|A^{N} p^{N} \phi-A \phi\right\|_{M^{2}} \leqq \gamma_{0} \rho(N)\left\|\phi^{1}\right\|_{W^{2,2}}, \quad N=1,2, \cdots,
$$

where $\gamma_{0}$ is a constant independent of $\phi$ and $N$. 
(c) If in addition $A_{01} \in W^{1,2}\left(-h_{i},-h_{i-1} ; \mathbb{R}^{n \times n}\right), i=1, \cdots, p$, then for any $\psi \in \tilde{D}^{*}$

$$
\begin{aligned}
& \left\|\left(A^{N}\right)^{*} p^{N} \psi-A^{*} \psi\right\|_{M^{2}} \\
& \quad \leqq \gamma_{0} \rho(N) \sum_{i=1}^{p}\left(\left\|\psi^{1}\right\|_{W^{2,2}\left(-h_{i},-h_{i-1} ; \mathbb{R}^{n}\right)}+\left\|A_{01}^{T} \psi^{0}\right\|_{W^{1,2}\left(-h_{i},-h_{i-1} ; \mathbb{R}^{n}\right)}\right),
\end{aligned}
$$

$N=1,2, \cdots$, where again $\gamma_{0}$ is a constant independent of $\phi$ and $N$.

Proof. (a) is obvious from (A). In order to prove (b), we put $\phi^{N}=\pi^{N} \phi^{1}$ and get, using (2.6) and Definition 5.2, for $\phi \in \tilde{D}$

$$
\begin{aligned}
& \left\|A^{N} p^{N} \phi-A \phi\right\|_{M^{2}} \\
& \leqq \sum_{i=1}^{p}\left|A_{i}\right|\left\|\phi^{N}-\phi^{1}\right\|_{L^{\infty}}+\left\|A_{01}\right\|_{L^{2}}\left\|\phi^{N}-\phi^{1}\right\|_{L^{2}} \\
& +\left\|\pi^{N}\left(\frac{d^{+}}{d \theta} \phi^{N}-\dot{\phi}^{1}\right)\right\|_{L^{2}}+\left\|\pi^{N}\left(\dot{\phi}^{1}\right)-\dot{\phi}^{1}\right\|_{L^{2}} \\
& +\left\|\delta_{0,-}^{N}\right\|\left|\phi^{0}-\lim _{\tau \uparrow 0} \phi^{N}(\tau)\right|+\sum_{i=1}^{p-1}\left\|\delta_{i,-}^{N}\right\|\left|\phi^{N}\left(-h_{i}\right)-\lim _{\tau \uparrow-h_{i}} \phi^{N}(\tau)\right| \\
& \leqq \sum_{i=1}^{p}\left|A_{i}\right|\left\|\phi^{N}-\phi^{1}\right\|_{L^{\infty}}+\left\|A_{01}\right\|_{L^{2}}\left\|\phi^{N}-\phi^{1}\right\|_{L^{2}} \\
& +\left\|\frac{d^{+}}{d \theta}\left(\phi^{N}-\phi^{1}\right)\right\|_{L^{2}}+\left\|\pi^{N}\left(\dot{\phi}^{1}\right)-\dot{\phi}^{1}\right\|_{L^{2}} \\
& +\kappa(N)\left\{\left|\phi^{1}(0)-\lim _{\tau \uparrow 0} \phi^{N}(\tau)\right|+\sum_{i=1}^{p-1}\left|\phi^{N}\left(-h_{i}\right)-\phi^{1}\left(-h_{i}\right)\right|+\sum_{i=1}^{p-1}\left|\phi^{1}\left(-h_{i}\right)-\lim _{\tau \uparrow-h_{i}} \phi^{N}(\tau)\right|\right\} \\
& \leqq\left[\sum_{i=1}^{p}\left|A_{i}\right|+(2 p-1) \kappa(N)\right]\left\|\phi^{N}-\phi^{1}\right\|_{L^{\infty}} \\
& +\left\|A_{01}\right\|_{L^{2}}\left\|\phi^{N}-\phi^{1}\right\|_{L^{2}}+\left\|\frac{d^{+}}{d \theta}\left(\phi^{N}-\phi^{1}\right)\right\|_{L^{2}}+\left\|\pi^{N}\left(\dot{\phi}^{1}\right)-\dot{\phi}^{1}\right\|_{L^{2}} \\
& \leqq \gamma_{0} \rho(N)\left\|\phi^{1}\right\|_{W^{2,2}}
\end{aligned}
$$

where in the last step we have used assumption (A). $\gamma_{0}$ is an appropriately chosen constant.

(c) Using (2.7), Lemma 5.3 and $\psi \in D\left(A^{*}\right)$, we get

$$
\begin{aligned}
\left\|\left(A^{N}\right)^{*} p^{N} \psi-A^{*} \psi\right\|_{M}^{2} \leqq \lim _{\tau \uparrow 0} & \psi^{N}(\tau)-\psi^{1}(0) \mid \\
& +\left\|\pi^{N}\left(A_{01}^{T} \psi^{0}-\frac{d^{+}}{d \theta} \psi^{N}\right)-A_{01}^{T} \psi^{0}+\frac{d^{+}}{d \theta} \psi^{1}\right\|_{L^{2}} \\
& +\sum_{i=1}^{p-1}\left\|\delta_{i,+}^{N}\right\|\left|A_{i}^{T} \psi^{0}+\lim _{\tau \uparrow-h_{i}} \psi^{N}(\tau)-\psi^{N}\left(-h_{i}\right)\right| \\
& +\left\|\delta_{p,+}^{N}\right\|\left|A_{p}^{T} \psi^{0}-\psi^{N}(-h)\right| \\
\leqq & (2 p-1) \kappa(N))\left\|\psi^{1}-\psi^{N}\right\|_{L^{\infty}} \\
& +\left\|\pi^{N}\left(A_{01}^{T} \psi^{0}-\frac{d^{+}}{d \theta} \psi^{1}\right)-\left(A_{01}^{T} \psi^{0}-\frac{d^{+}}{d \theta} \psi^{1}\right)\right\|_{L^{2}} \\
& +\left\|\frac{d^{+}}{d \theta}\left(\psi^{1}-\psi^{N}\right)\right\|_{L^{2}}
\end{aligned}
$$


Under the given assumptions we have $\psi^{1} \in W^{2,2}\left(-h_{i},-h_{i-1} ; \mathbb{R}^{n}\right), i=1, \cdots, p$. Therefore we get from (A), (ii)

$$
\begin{aligned}
& \left\|\pi^{N}\left(A_{01}^{T} \psi^{0}-\frac{d^{+}}{d \theta} \psi^{1}\right)-\left(A_{01}^{T} \psi^{0}-\frac{d^{+}}{d \theta} \psi^{1}\right)\right\|_{L^{2}} \\
& \leqq \rho(N) \sum_{i=1}^{p}\left\|A_{01}^{T} \psi^{0}-\frac{d^{+}}{d \theta} \psi^{1}\right\|_{W^{1,2}\left(-h_{i},-h_{i-1} ; \mathbb{R}^{n}\right)} \\
& \leqq \rho(N) \sum_{i=1}^{p}\left(\left\|A_{01}^{T} \psi^{0}\right\|_{W^{1,2}\left(-h_{i},-h_{i-1} ; \mathbb{R}^{n}\right)}+\left\|\psi^{1}\right\|_{W^{2,2}\left(-h_{i},-h_{i-1} ; \mathbb{R}^{n}\right)}\right)
\end{aligned}
$$

and

$$
\begin{aligned}
\left\|\left(A^{N}\right)^{*} p^{N} \psi-A^{*} \psi\right\|_{M^{2}} \leqq \gamma_{0} \rho(N) \sum_{i=1}^{p}( & \left\|A_{01}^{T} \psi^{0}\right\|_{W^{1,2}\left(-h_{i},-h_{i-1} ; \mathbb{R}^{n}\right)} \\
& \left.+\left\|\psi^{1}\right\|_{W^{2,2}\left(-h,-h_{i-1} ; \mathbb{R}^{n}\right)}\right) .
\end{aligned}
$$

It is clear that under the conditions of Lemma 5.5, hypothesis (H3), (i) is satisfied for $X^{N}, p^{N}, A^{N}, N=1,2, \cdots$, if we take $D=\operatorname{dom} A^{2}$, and for $X^{N}, p^{N},\left(A^{N}\right)^{*}$, $N=1,2, \cdots$, if we take $D=\operatorname{dom}\left(A^{*}\right)^{2}$. The next lemma establishes (H3), (ii).

LemMA 5.6. Assume that (A) is satisfied.

(a) There exist constants $\tilde{M} \geqq 1$ and $\omega \in \mathbb{R}$ such that for all $\phi \in \operatorname{dom} A^{2}$

$$
\left\|A^{N} p^{N} S(t) \phi\right\|_{M^{2}} \leqq \tilde{M} e^{\omega t}|\phi|_{2}, \quad t \geqq 0, \quad N=1,2, \cdots,
$$

where $|\phi|_{2}=\|\phi\|+\|A \phi\|+\left\|A^{2} \phi\right\|$.

(b) If in addition $A_{01} \in W^{1,2}\left(-h_{i},-h_{i-1} ; \mathbb{R}^{n \times n}\right), i=1, \cdots, p$, then there exist constants $M^{*} \geqq 1$ and $\omega \in \mathscr{R}$ such that for all $\psi \in \operatorname{dom}\left(A^{*}\right)^{2}$

$$
\left\|\left(A^{N}\right)^{*} p^{N} S^{*}(t) \psi\right\|_{M^{2}} \leqq M^{*} e^{\omega t}|\psi|_{2}^{*}, \quad t \geqq 0, \quad N=1,2, \cdots,
$$

where $|\psi|_{2}^{*}=\|\psi\|+\left\|A^{*} \psi\right\|+\left\|\left(A^{*}\right)^{2} \psi\right\|$.

Proof. (a) Since $S(\cdot)$ restricted to dom $A^{2}$ is a $C_{0}$-semigroup on dom $A^{2}$ equipped with the graph norm $|\cdot|_{2}$, we have

$$
|S(t) \phi|_{2} \leqq M e^{\omega t}|\phi|_{2}, \quad t \geqq 0, \quad \phi \in \operatorname{dom} A^{2},
$$

with some constants $M \geqq 1, \omega \in \mathbb{R}$. From

$$
A^{k} \phi=\left(L\left(\frac{d^{k-1}}{d \theta^{k-1}} \phi^{1}\right), \frac{d^{k}}{d \theta^{k}} \phi^{1}\right),
$$

$k=1,2, \cdots, \phi \in \operatorname{dom} A^{k}$, we see that

$$
\left\|\phi^{1}\right\|_{W^{2,2}} \leqq|\phi|_{2}, \quad \phi \in \operatorname{dom} A^{2} .
$$

Therefore, for $\phi \in \operatorname{dom} A^{2}$

$$
\left\|(S(t) \phi)^{1}\right\|_{W^{2,2} \leqq M} e^{\omega t}|\phi|_{2}, \quad t \geqq 0
$$

and by Lemma 5.5, (b)

$$
\begin{aligned}
\left\|A^{N} p^{N} S(\phi)\right\| & \leqq\|A S(t) \phi\|+\left\|A^{N} p^{N} S(t) \phi-A S(t) \phi\right\| \\
& \leqq M e^{\omega t}\|A \phi\|+\gamma_{0} \rho(N) M e^{\omega t}|\phi|_{2} \\
& \leqq M\left(1+\gamma_{0} \rho(N)\right) e^{\omega t}|\phi|_{2}, \quad t \geqq 0, \quad N=1,2, \cdots .
\end{aligned}
$$


(b) As in part (a) we have

$$
\left|S^{*}(t) \psi\right|_{2}^{*} \leqq M e^{\omega t}|\psi|_{2}^{*}, \quad t \geqq 0, \quad \psi \in \operatorname{dom}\left(A^{*}\right)^{2} .
$$

Using (2.7), $\left(A^{*}\right)^{2} \psi=\left(\cdots, A_{01}^{T}\left(\psi^{1}(0)+A_{0}^{T} \psi^{0}\right)-d^{+} / d \theta\left(A_{01}^{T} \psi^{0}-d^{+} / d \theta \psi^{1}\right)\right)$ and $d^{+} / d \theta\left(A_{01}^{T} \psi^{0}-d^{+} / d \theta \psi^{1}\right)=\dot{A}_{01}^{T} \psi^{0}-\ddot{\psi}^{1}$ on the intervals $\left[-h_{i},-h_{i-1}\right), i=1, \cdots, p$, it is not difficult to see that for a constant $\kappa=\kappa\left(\left\|A_{01}\right\|_{L^{2}},\left\|d^{+} / d \theta A_{01}\right\|_{L^{2}}\right)$ the following estimate is valid:

$$
\sum_{i=1}^{p}\left\|\psi^{1}\right\|_{W^{2,2}\left(-h,-h_{i-1} ; \mathbb{R}^{n}\right)} \leqq \kappa|\psi|_{2}^{*}, \quad \psi \in \operatorname{dom}\left(A^{*}\right)^{2} .
$$

The rest of the proof is analogous to that for part (a) but now using Lemma 5.5, (c).

From Lemmas 5.4-5.6 it immediately follows that under the assumption specified in these lemmas Theorem 4.1 applies to the sequences $X^{N}, p^{N}, A^{N},\left(A^{N}\right)^{*}, N=1,2$, $3, \cdots$, defined in this section. The approximating control systems on $X^{N}$ are given by (compare (4.5) and (4.9))

$$
\begin{aligned}
& \dot{w}^{N}(t)=A^{N} w^{N}(t)+B^{N} u(t), \\
& y^{N}(t)=C^{N} w^{N}(t), \quad t \geqq 0, \\
& w^{n}(0)=p^{N} \phi, \quad \phi \in M^{2},
\end{aligned}
$$

with the cost functional

$$
J^{N}(u)=\left\langle w^{N}(T), G w^{N}(T)\right\rangle+\int_{0}^{T}\left[\left|y^{N}(t)\right|^{2}+u(t)^{T} R u(t)\right] d t,
$$

where the input and output operators are given by

$$
\begin{aligned}
& B^{N} u=p^{N} B u=B u, \quad u \in \mathbb{R}^{l}, \\
& C^{N} \phi=C p^{N} \phi=C \phi, \quad \phi \in X^{N} .
\end{aligned}
$$

As in $\S 3.2$ we assume that $R \in \mathbb{R}^{l \times l}$ is positive definite, $G_{0} \in \mathbb{R}^{n \times n}$ is positive semidefinite and $G: M^{2} \rightarrow M^{2}$ is defined by $G \phi=\left(G_{0} \phi^{0}, 0\right)$. The Riccati operators corresponding to $\left(\Sigma^{N}\right)$ and $(5.8)$ satisfy

$$
\Pi^{N}(t)=p^{N} \Pi(t)^{N} p^{N}, \quad 0 \leqq t \leqq T,
$$

and (restricted to $X^{N}$ )

$$
\begin{aligned}
& \frac{d}{d t} \Pi^{N}(t)+\left(A^{N}\right)^{*} \Pi^{N}(t)+\Pi^{N}(t) A^{N} \\
& \quad-\Pi^{N}(t) B^{N} R^{-1}\left(B^{N}\right) * \Pi^{N}(t)+\left(C^{N}\right)^{*} C^{N}=0, \quad 0 \leqq t \leqq T, \\
& \Pi^{N}(T)=G^{N} .
\end{aligned}
$$

Here $G^{N}$ is the restriction of $G$ to $X^{N}$.

It follows from the results of this section that Theorem 4.3 applies to system $\left(\Sigma^{N}\right)$ with (5.8). More precisely, we have the following theorem which may be considered as the main result of this paper.

THEOREM 5.7. Let hypothesis (A) be satisfied and assume that $A_{01} \in$ $W^{1,2}\left(-h_{i},-h_{i-1} ; \mathbb{R}^{n \times n}\right)$ for $i=1, \cdots, p$. Then

$$
\lim _{N \rightarrow \infty}\left\|\Pi(t)-\Pi^{N}(t)\right\|=0
$$

uniformly for $0 \leqq t \leqq T$. 
Of course, also assertions (a)-(c) of Theorem 4.3 are true for system $\left(\Sigma^{N}\right),(5.8)$ and $(\Sigma),(3.1)$ (or equivalently $(2.1),(3.1)$ ).

5.2. Matrix representations. For implementation of a scheme obtained along the lines described in the previous section we have to compute matrix representations $\left[A^{N}\right],\left[\left(A^{N}\right)^{*}\right],\left[B^{N}\right],\left[C^{N}\right]$ and $\left[G^{N}\right]$ of the operators $A^{N},\left(A^{N}\right)^{*}, B^{N}, C^{N}$ and $G^{N}$, respectively, with respect to the basis $\hat{E}^{N}$. How to compute the coordinate vector of $p^{N} \phi$ for $\phi \in M^{2}$ has already been shown (see (5.2)).

Define the $k_{N} \times k_{N}$-matrices $h_{i}^{N}, i=1, \cdots, p$, and $g_{i}^{N}, i=1, \cdots, p-1$, by

$$
\begin{aligned}
& h_{i}^{N}=\left(\left\langle e_{i l}^{N}, \frac{d^{+}}{d \theta} e_{i m}^{N}\right\rangle_{L^{2}}-\lim _{\tau \uparrow-h_{i-1}} e_{i l}^{N}(\tau) e_{i m}^{N}(\tau)\right)_{l, m=1, \cdots, k_{N}}, \\
& g_{i}^{N}=\left(\lim _{\tau \uparrow-h_{i}} e_{i+1, l}^{N}(\tau) e_{i, m}^{N}\left(-h_{i}\right)\right)_{l, m=1, \cdots, k_{N}}
\end{aligned}
$$

and put

$$
\begin{aligned}
& A_{i j}^{N}=\left\langle A_{01}, e_{i j}^{N} I\right\rangle_{L^{2}}, \quad i=1, \cdots, p, \quad j=1, \cdots, k_{N}, \\
& \alpha_{i}^{N}=\left(A_{i} e_{i 1}^{N}\left(-h_{i}\right)+A_{i 1}^{N}, \cdots, A_{i} e_{i k_{N}}^{N}\left(-h_{i}\right)+A_{i k_{N}}^{N}\right) \in \mathbb{R}^{n \times n k_{N}},
\end{aligned}
$$

$i=1, \cdots, p$, and

$$
\beta^{N}=\operatorname{col}\left(\lim _{\tau \uparrow 0} e_{11}^{N}(\tau) I, \cdots, \lim _{\tau \uparrow 0} e_{1 k_{N}}^{N}(\tau) I\right) \in \mathbb{R}^{n k_{N} \times n} .
$$

Furthermore, define the $\left(p k_{N}+1\right) n \times\left(p k_{N}+1\right) n$-matrix $H^{N}$ by

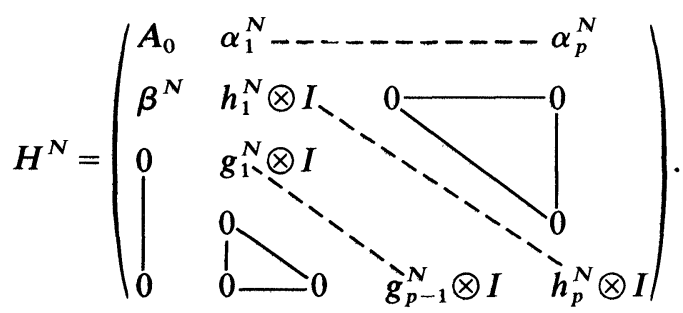

Proposition 5.8. (a) $\left[A^{N}\right]=\left(Q^{N}\right)^{-1} H^{N}$.

(b) $\quad\left[\left(A^{N}\right)^{*}\right]=\left(Q^{N}\right)^{-1}\left(H^{N}\right)^{T}$.

(c) $\quad\left[B^{N}\right]=\operatorname{col}\left(B_{0}, 0, \cdots, 0\right) \in \mathbb{R}^{n\left(p k_{N} \times 1\right) \times l}$.

(d) $\left[C^{N}\right]=\left(C_{0}, 0, \cdots, 0\right) \in \mathbb{R}^{m \times n\left(p k_{N}+1\right)}$.

(e)

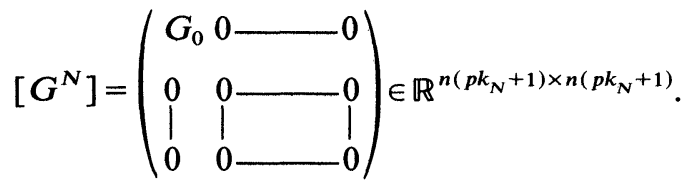

Proof. $\left[A^{N}\right]$ is characterized by

$$
\alpha^{N}\left(A^{N} \phi\right)=\left[A^{N}\right] \alpha^{N}(\phi), \quad \phi \in X^{N} .
$$

On the other hand we get from (5.2) and $\phi=\hat{E}^{N} \alpha^{N}(\phi)$

$$
\alpha^{N}\left(A^{N} \phi\right)=\left(Q^{N}\right)^{-1} d^{N}\left(A^{N} \phi\right)=\left(Q^{N}\right)^{-1}\left\langle\hat{E}^{N}, A^{N} \hat{E}^{N}\right\rangle \alpha^{N}(\phi),
$$

i.e.,

$$
\left[A^{N}\right]=\left(Q^{N}\right)^{-1}\left\langle\hat{E}^{N}, A^{N} \hat{E}^{N}\right\rangle
$$


From Definition 5.2 we get

$$
\begin{aligned}
A^{N} \hat{e}_{0}^{N}=\left(A_{0}, 0\right)+\delta_{0,-}^{N}, & \\
A^{N} \hat{e}_{i j}^{N}= & \left(A_{i} e_{i j}^{N}\left(-h_{i}\right)+A_{i j}^{N}, \pi^{N}\left(\frac{d^{+}}{d \theta} e_{i j}^{N}\right)\right) \\
& \quad-\delta_{i-1,-}^{N} \lim _{\tau \uparrow-h_{i-1}} e_{i j}^{N}(\tau)+\delta_{i,-}^{N} e_{i j}^{N}\left(-h_{i}\right),
\end{aligned}
$$

$i=1, \cdots, p-1, j=1, \cdots, k_{N}$, and

$$
\begin{aligned}
A^{N} \hat{e}_{p j}^{N}=\left(A_{p} e_{p j}^{N}(-h)+A_{p j}^{N}, \pi^{N}\left(\frac{d^{+}}{d \theta} e_{p j}^{N}\right)\right) & \\
& -\delta_{p-1,-}^{N} \lim _{\tau \uparrow-h_{p-1}} e_{p j}^{N}(\tau), \quad j=1, \cdots, k_{N}
\end{aligned}
$$

Observing

$$
\left\langle e_{i l}^{N}, \pi^{N}\left(\frac{d^{+}}{d \theta} e_{k m}^{N}\right)\right\rangle_{L^{2}}=\left\langle e_{i l}^{N}, \frac{d^{+}}{d \theta} e_{k m}^{N}\right\rangle_{L^{2}},
$$

Lemma 5.1 and (5.2) we obtain by straightforward calculation

$$
\left\langle\hat{E}^{N}, A^{N} \hat{E}^{N}\right\rangle=H^{N} \text {. }
$$

In order to prove the representation for $\left[\left(A^{N}\right)^{*}\right]$, we use (5.3) and get

$$
\begin{aligned}
\alpha^{N}(\phi)^{T} Q^{N}\left[\left(A^{N}\right)^{*}\right] \alpha^{N}(\psi) & =\alpha^{N}(\phi)^{T} Q^{N} \alpha^{N}\left(\left(A^{N}\right)^{*} \psi\right) \\
& =\left\langle\phi,\left(A^{N}\right)^{*} \psi\right\rangle=\left\langle A^{N} \phi, \psi\right\rangle=\alpha^{N}\left(A^{N} \phi\right)^{T} Q^{N} \alpha^{N}(\psi) \\
& =\alpha^{N}(\phi)^{T}\left[A^{N}\right]^{T} Q^{N} \alpha^{N}(\psi)=\alpha^{N}(\phi)^{T}\left(H^{N}\right)^{T} \alpha^{N}(\psi)
\end{aligned}
$$

for all $\phi, \psi \in X^{N}$, i.e.,

$$
\left[\left(A^{N}\right)^{*}\right]=\left(Q^{N}\right)^{-1}\left(H^{N}\right)^{T}
$$

For $u \in \mathbb{R}^{l}$ we have

$$
\begin{aligned}
\alpha^{N}\left(B^{N} u\right) & =\alpha^{N}\left(\left(B_{0} u, 0\right)\right)=\left(Q^{N}\right)^{-1} d^{N}\left(\left(B_{0} u, 0\right)\right) \\
& =\left(Q^{N}\right)^{-1} \operatorname{col}\left(B_{0} u, 0, \cdots, 0\right)=\operatorname{col}\left(B_{0}, 0, \cdots, 0\right) u,
\end{aligned}
$$

which proves the given form of $\left[B^{N}\right]$. The proofs for $\left[C^{N}\right]$ and $\left[G^{N}\right]$ are analogous.

It is obvious from Proposition 5.8 that $\left[\left(A^{N}\right)^{*}\right]=\left(Q^{N}\right)^{-1}\left[A^{N}\right] Q^{N}$. Therefore we do not get the standard Riccati matrix differential equation if we take everywhere in (5.9) the matrix representations of the operators involved. In order to overcome this difficulty we define

$$
\Gamma^{N}(t)=Q^{N}\left[\Pi^{N}(T-t)\right], \quad 0 \leqq t \leqq T,
$$

and get from (5.9) the standard Riccati equation for $\Gamma^{N}(t)$

$$
\begin{aligned}
\frac{d}{d t} \Gamma^{N}= & {\left[A^{N}\right]^{T} \Gamma^{N}+\Gamma^{N}\left[A^{N}\right] } \\
& -\Gamma^{N}\left[B^{N}\right] R^{-1}\left[B^{N}\right]^{T} \Gamma^{N}+\left[C^{N}\right]^{T}\left[C^{N}\right], \quad 0 \leqq t \leqq T, \\
\Gamma^{N}(0)= & {\left[G^{N}\right] . }
\end{aligned}
$$

Note, that $\left[\left(B^{N}\right)^{*}\right]=\left[B^{N}\right]^{T},\left[\left(C^{N}\right)^{*}\right]=\left[C^{N}\right]^{T}$ and that $\Pi^{N}(t)^{*}=\Pi^{N}(t)$ implies $\Gamma^{N}(t)^{T}=\Gamma^{N}(t)$. 
Equation (5.11) can advantageously be solved using a method due to Casti and Kailath (see for instance [35, pp. $304 \mathrm{ff}$.]) which we indicate here for $p=1$ and $A_{01} \equiv 0$. We define

$$
W_{0}=A_{0}^{T} G_{0}+G_{0} A-G_{0} B_{0} R^{-1} B_{0}^{T} G_{0}+C_{0}^{T} C_{0}
$$

and the $2 n \times\left(k_{N}+1\right) n$-matrices

$$
F_{1}^{N}=\left(\begin{array}{ccc}
W_{0} & 0-0 & G_{0} A_{1} \\
A_{1}^{T} G_{0} & 0-0
\end{array}\right), \quad F_{2}^{N}=\left(\begin{array}{ccc}
I & 0-0 \\
0-0 & I
\end{array}\right) .
$$

Then

$$
\Gamma^{N}(t)=\left[G^{N}\right]+\int_{0}^{t} L_{1}^{N}(\tau)^{T} L_{2}^{N}(\tau) d \tau
$$

where

$$
\begin{aligned}
& \frac{d}{d t} L_{i}^{N}(t)=L_{i}^{N}(t)\left(\left[A^{N}\right]-\left[B^{N}\right] R^{-1}\left[B^{N}\right]^{T} \Gamma^{N}(t)\right), \\
& L_{i}^{N}(0)=F_{i}^{N}, \quad i=1,2 .
\end{aligned}
$$

Note that this is a system of $4 n^{2}\left(k_{N}+1\right)$ differential equations compared to the $n^{2}\left(k_{N}+1\right)^{2}$ differential equations of system (5.11) (in case $p=1, A_{01} \equiv 0$ ).

If in the optimal feedback law (3.13) for the delay system $(2.1)$ we use $\Pi^{N}(t)$ instead of $\Pi(t)$ we get the suboptimal controls (compare (4.17))

$$
\hat{u}^{N}(t)=-R^{-1} B^{*} \Pi^{N}(t) p^{N}\left(\hat{x}^{N}(t), \hat{x}_{t}^{N}\right), \quad 0 \leqq t \leqq T,
$$

where $\hat{x}^{N}(t)$ is the solution of $(2.1)$ with $u(t)=\hat{u}^{N}(t)$. We introduce the $n \times n$-matrices $\Pi_{0}^{N}(t), \Pi_{11}^{N}(t), \cdots, \Pi_{p k_{N}}^{N}(t)$ by

$$
\Pi^{N}(t)=\left(\left.\left.\begin{array}{l}
\Pi_{0}^{N}(t) \\
\Pi_{11}^{N}(t) \\
\vdots \\
\Pi_{p k_{N}}^{N}(t)
\end{array}\right|_{* L^{*}} ^{*}\right|^{*}\right.
$$

and define

$$
\Pi_{1}^{N}(t, \tau)=\sum_{i=1}^{p} \sum_{j=1}^{k_{N}} \Pi_{i j}^{N}(t)^{T} e_{i j}^{N}(\tau)
$$

for $-h \leqq \tau \leqq 0$ and $0 \leqq t \leqq T$. Then the control law (5.12) takes the following form:

$$
\begin{aligned}
& \hat{u}^{N}(t)=-R^{-1}\left[B^{N}\right]^{T}\left[\Pi^{N}(t)\right]\left(Q^{N}\right)^{-1} d^{N}\left(\left(\hat{x}^{N}(t), \hat{x}_{t}^{N}\right)\right) \\
&=-R^{-1}\left(B_{0}^{T}, 0, \cdots, 0\right)\left[\Pi^{N}(t)\right]^{T} d^{N}\left(\left(\hat{x}^{N}(t), \hat{x}_{t}^{N}\right)\right) \\
&=-R^{-1} B_{0}^{T}\left\{\Pi_{0}^{N}(t)^{T} \hat{x}^{N}(t)\right.\left.\quad+\sum_{i=1}^{p} \sum_{j=1}^{k_{N}} \Pi_{i j}^{N}(t)^{T} \int_{-h}^{0} e_{i j}^{N}(\tau) \hat{x}^{N}(t+\tau) d \tau\right\} \\
&=-R^{-1} B_{0}^{T}\left\{\Pi_{0}^{N}(t) \hat{x}^{N}(t)+\int_{-h}^{0} \Pi_{1}^{N}(t, \tau) \hat{x}^{N}(t+\tau) d \tau\right\} .
\end{aligned}
$$


The additional condition used in [20] in order to prove convergence results analogous to those contained in Theorem 4.3 for the infinite time horizon problem in general cannot be satisfied for concrete realizations of the approximation scheme presented in this section. Especially this condition is not satisfied for the concrete realization of the scheme using spline functions as described in the next section. Despite this fact we did also numerical computations using the spline scheme for the infinite time horizon problem (see $\S 6.2$ ). Therefore we conclude this section with a short description of the equations governing the approximation of this problem. Consider system $\left(\Sigma^{N}\right)$ with the cost functional

$$
J^{N}(u)=\int_{0}^{\infty}\left[\left|y^{N}(t)\right|^{2}+u(t)^{T} R u(t)\right] d t
$$

The feedback law which minimizes $J^{N}(u)$ subject to $\left(\Sigma^{N}\right)$ is governed by the Riccati operator $\Pi^{N}$ which satisfies $\Pi^{N}=p^{N} \Pi^{N} p^{N}$ and (restricted to $X^{N}$ ) the algebraic Riccati equation

$$
\left(A^{N}\right) * \Pi^{N}+\Pi^{N} A^{N}-\Pi^{N} B R^{-1} B^{*} \Pi^{N}+C^{*} C=0 .
$$

Analogously to (5.10) we define $\Gamma^{N}=Q^{N}\left[\Pi^{N}\right]$ and obtain the standard algebraic Riccati matrix equation

$$
\left[A^{N}\right]^{T} \Gamma^{N}+\Gamma^{N}\left[A^{N}\right]-\Gamma^{N}\left[B^{N}\right] R^{-1}\left[B^{N}\right]^{T} \Gamma^{N}+\left[C^{N}\right]^{T}\left[C^{N}\right]=0 .
$$

Note, that as for the finite time horizon problem, $\Gamma^{N}=\left(\Gamma^{N}\right)^{T}$ follows from $\Pi^{N}=\left(\Pi^{N}\right)^{*}$. Using $\Pi^{N}$ instead of $\Pi$ in the feedback law (3.16) we get by analogous computations as in (5.15) the suboptimal control law

$$
\hat{u}^{N}=-R^{-1} B_{0}^{T}\left\{\Pi_{0}^{N} \hat{x}^{N}(t)+\int_{-h}^{0} \Pi_{1}^{N}(\tau) \hat{x}^{N}(t+\tau) d \tau\right\}, \quad t \geqq 0,
$$

where $\Pi_{1}^{N}(\tau)=\sum_{i=1}^{p} \sum_{j=1}^{k_{N}}\left(\Pi_{i j}^{N}\right)^{T} e_{i j}(\tau)$ and the $n \times n$-matrices $\Pi_{0}^{N}, \Pi_{i j}^{N}, i=1, \cdots, p$, $j=1, \cdots, k_{N}$, are defined by

$$
\left[\Pi^{N}\right]=\left(\left.\begin{array}{ll}
\Pi_{0}^{N} & * \\
\Pi_{11}^{N} & \\
\vdots & \\
\Pi_{p k_{N}}^{N} & *
\end{array}\right|_{*} ^{*}\right.
$$

(compare (5.13) and (5.14)).

5.3. The spline scheme. In this section we give a realization of the scheme developed in $\S 5.1$ by using first order spline functions.

For $N=1,2, \cdots$ we choose the meshpoints

$$
t_{i j}^{N}=-h_{i-1}-j \frac{r_{i}}{N}, \quad i=1, \cdots, p, \quad j=0, \cdots, N,
$$

where $r_{i}=h_{i}-h_{i-1}$ and define the basis splines

$$
e_{i 0}^{N}(\tau)= \begin{cases}\frac{N}{r_{i}}\left(\tau-t_{i 1}^{N}\right) & \text { for } t_{i 1}^{N} \leqq \tau<t_{i 0}^{N} \\ 0 & \text { elsewhere }\end{cases}
$$




$$
e_{i j}^{N}(\tau)= \begin{cases}-\frac{N}{r_{i}}\left(\tau-t_{i, j-1}^{N}\right) & \text { for } t_{i j}^{N} \leqq \tau<t_{i, j-1}^{N}, \\ \frac{N}{r_{i}}\left(\tau-t_{i, j+1}^{N}\right) & \text { for } t_{i, j+1}^{N} \leqq \tau<t_{i, j}^{N}, \\ 0 & \text { elsewhere, }\end{cases}
$$

$j=1, \cdots, N-1$, and

$$
e_{i N}^{N}(\tau)= \begin{cases}-\frac{N}{r_{i}}\left(\tau-t_{i, N-1}^{N}\right) & \text { for } t_{i N}^{N} \leqq \tau<t_{i, N-1}^{N}, \\ 0 & \text { elsewhere, }\end{cases}
$$

$i=1, \cdots, p$. Note, that compared to $\S 5.1$ we slightly have changed the enumeration of the $e_{i j}^{N}$ ( $j$ running from 0 to $N$ now). We have $k_{N}=N+1$ and therefore $\operatorname{dim} X^{N}=$ $(p(N+1)+1) n$.

An easy computation shows that the matrices $q_{i}^{N}$ which determine $Q^{N}$ (recall $Q^{N}=\operatorname{diag}\left(I, q_{1}^{N} \otimes I, \cdots, q_{p}^{N} \otimes I\right)$ are given by $q_{i}^{N}=\left(r_{i} / N\right) q^{N}$, where

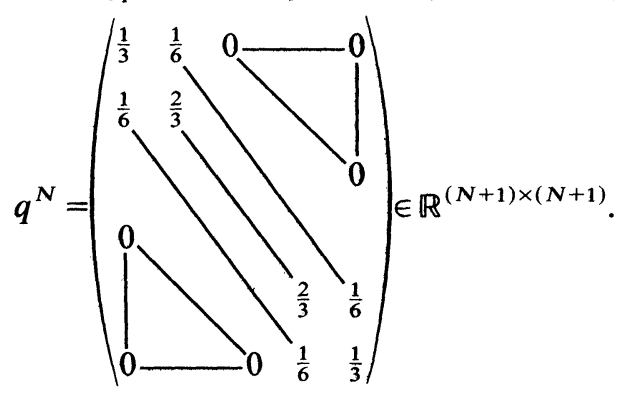

For the approximating delta impulses we have

LeMmA 5.9. For all $N=1,2, \cdots$,

$$
\begin{aligned}
\left\|\delta_{i,+}^{N}\right\| \leqq\left(\frac{6 N}{r_{i}}\right)^{1 / 2}, & i=1, \cdots, p \\
\left\|\delta_{i,-}^{N}\right\| \leqq\left(\frac{6 N}{r_{i+1}}\right)^{1 / 2}, & i=0, \cdots, p-1 .
\end{aligned}
$$

Proof. Using (5.3) and the definition of $\delta_{i,+}^{N}$ and $Q^{N}$ we get for any $x \in \mathbb{R}^{n}$

$$
\begin{aligned}
\left\|\delta_{i,+}^{N} x\right\|^{2} & =\left(\gamma_{i,+}^{N} x\right)^{T} Q^{N}\left(Q^{N}\right)^{-1} Q^{N} \gamma_{i,+}^{N} x \\
& =\frac{N}{r_{i}} x^{T}(0, \cdots, 0, I)\left(q^{N} \otimes I\right)^{-1} \operatorname{col}(0, \cdots, 0, I) x \\
& \leqq \frac{6 N}{r_{i}}|x|^{2},
\end{aligned}
$$

where we have used $\lambda_{\min }\left(q^{N}\right) \geqq 1 / 6$. The estimate for $\delta_{i,-}^{N}$ is analogous.

As a consequence of Lemma 5.9 we have

$$
\kappa(N) \leqq\left(\frac{6 N}{\rho}\right)^{1 / 2} \quad \text { with } \rho=\min _{i=1, \cdots, p} r_{i} .
$$

Proposition 5.10. Hypothesis (A) is satisfied for the spline scheme with $\rho(N)=$ const/ N. As a consequence Theorem 5.7 is valid for the spline scheme. 
Proof. The following estimates are standard estimates for spline functions:

$$
\begin{aligned}
& \left\|f-\pi_{i}^{N} f\right\|_{L^{2}} \leqq \frac{\text { const }}{N^{2}}\|\ddot{f}\|_{L^{2}}, \\
& \left\|\frac{d}{d \theta}\left(f-\pi_{i}^{N} f\right)\right\|_{L^{2}} \leqq \frac{\text { const }}{N}\|\ddot{f}\|_{L^{2}}
\end{aligned}
$$

for $f \in W^{2,2}\left(-h_{i},-h_{i-1} ; \mathbb{R}^{n}\right)($ see $[39$, Thm. 6.5]) and

$$
\left\|f-\pi_{i}^{N} f\right\|_{L^{2}} \leqq \frac{\text { const }}{N}\|\dot{f}\|_{L^{2}}
$$

for $f \in w^{1,2}\left(-h_{i},-h_{i-1} ; \mathbb{R}^{n}\right)$ (see [39, Exercise 6.1]). In order to get the estimate for the $L^{\infty}$-norm, we observe that $\pi_{i}^{N} f=\ddot{\chi}^{N}$, where $\chi^{N}$ is the cubic type $I$ interpolating spline for $\Phi(\tau)=\int_{-h_{i}}^{\tau} \int_{-h_{i}}^{\theta} f(\sigma) d \sigma d \theta, \tau \in\left[-h_{i},-h_{i-1}\right]$ (see, for instance, [39, Proof of Thm. 6.6]). Note, that interpolating cubic splines in [39] are always type $I$ interpolating splines in the terminology of [1]. Then we, get from [23, Thm. 5.7.1] (with $L=d^{2} / d \theta^{2}$ and $m=2$ ) or $[11$, p. 235] (with $m=r=2, q=\infty$ )

$$
\left\|f-\pi_{i}^{N} f\right\|_{L^{\infty}} \leqq \text { const }\left(\frac{1}{N}\right)^{3 / 2}\|f\|_{W^{2,2}}
$$

for $f \in W^{2,2}\left(-h_{i},-h_{i-1} ; \mathbb{R}^{n}\right)$. These estimates together with Lemma 5.9 imply (A).

Using $e_{i, j}^{N}\left(-h_{i}\right)=1$ for $j=N$ and $=0$ for $j=0, \cdots, N-1, \lim _{\tau \uparrow-h_{i}} e_{i+1, j}^{N}(\tau)=1$ for $j=0$ and $=0$ for $j=1, \cdots, N$, we immediately get

$$
\begin{aligned}
& \alpha_{i}^{N}=\left(A_{i 0}^{N}, \cdots, A_{i, N-1}^{N}, A_{i N}^{N}+A_{i}\right), \quad i=1, \cdots, p, \\
& \beta^{N}=\operatorname{col}(I, 0, \cdots, 0) \text {, }
\end{aligned}
$$

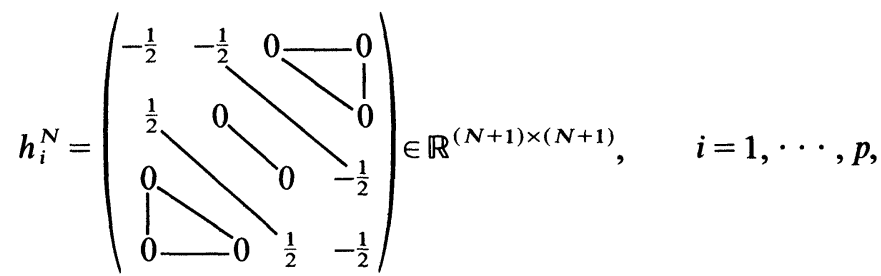

and

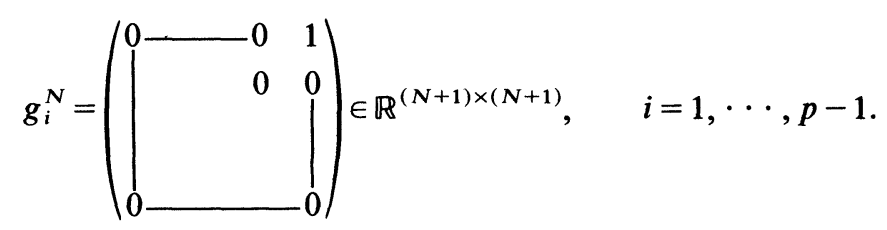

Recall Proposition 5.8 for the matrix representations of $A^{N}$ and $\left(A^{N}\right)^{*}$.

We conclude this section with some remarks:

(1) As we already mentioned in the Introduction, the spline scheme developed in this paper has interesting qualitative properties. For instance the relations between the state concept as defined in (2.4) and the dual state concept which are governed by the so called structural operator $F$ are preserved under approximation (see [10], [13], [16], [17], [28]; [31]). These results will be published elsewhere (see also [26]). 
(2) Another very important property of the spline scheme is that the approximating systems $\left(\Sigma^{N}\right)$ are stable, stabilizable or detectable for all $N$ sufficiently large whenever the delay system has the property (see [26]).

(3) In order to use the results of [20] for the infinite time horizon problem, the approximation scheme should have the following property: If the delay system (2.1) is stable, i.e. $\|S(t)\| \leqq M e^{-\varepsilon t}, t \geqq 0$, with $M \geqq 1, \varepsilon>0$, then there exist constants $\tilde{M} \geqq 1$ and $\tilde{\varepsilon}>0$ such that for all $N$ sufficiently large

$$
\left\|S^{N}(t)\right\| \leqq \tilde{M} e^{-\tilde{\varepsilon} t}, \quad t \geqq 0 .
$$

It can be shown that this property is not satisfied for our spline scheme (see [26] for details).

(4) The assumption $e_{i j}^{N} \mid\left[-h_{i},-h_{i-1}\right) \in W^{1,2}\left(-h_{i},-h_{i-1} ; \mathbb{R}\right)$ can easily be weakened. One can allow jumps of $e_{i j}^{N}$ in the interval $\left[-h_{i},-h_{i-1}\right)$. Then the definition of $A^{N}$ has to be modified by adding additional terms containing the approximating delta impulses corresponding to these additional jumps. This idea has been used in [34], where a realization of the scheme using piecewise linear functions is investigated. If one uses stepfunctions then one obtains the well-known averaging scheme.

(5) An important feature of the scheme developed in $\S 5.1$ is that due to the product space structure of $X^{N}$ orthogonality of the functions $e_{i j}^{N}, i=1, \cdots, p, j=$ $1, \cdots k_{N}$, implies orthogonality of the "basis elements"

$$
\hat{e}_{0}^{N}, \hat{e}_{i j}^{N}, \quad i=1, \cdots, p, \quad j=1, \cdots, k_{N} .
$$

This property has been exploited in [25] where a very efficient realization of the scheme by using Legendre polynomials is discussed.

6. Numerical results for the optimal control problem. The spline algorithm presented in $\S 5.3$ was applied to a large number of examples. In this section we present the numerical findings for some of those examples. The numerical results confirm the theoretical results in case of the finite time horizon problem. The scheme performs also very well in case of the infinite time horizon problem. This is shown by two examples which already have been considered in the literature [8].

6.1. An example with finite final time. This is the problem of minimizing

$$
J(u)=\frac{3}{2} x(3)^{2}+\frac{1}{2} \int_{0}^{3} u(t)^{2} d t
$$

subject to

$$
\begin{gathered}
\dot{x}(t)=x(t-1)+u(t), \quad 0 \leqq t \leqq T=3, \\
\phi^{0}=\phi^{1}(0), \quad \phi^{1}(t) \equiv 1 .
\end{gathered}
$$

For this example we have $n=1, p=1, A_{01} \equiv 0, A_{0}=C_{0}=0, A_{1}=B_{0}=1, G_{0}=\frac{3}{2}$ and $R=\frac{1}{2}$. The optimal controls, trajectories and costs were calculated in [5] using the maximum principle and are given by

$$
\bar{u}(t)=\left\{\begin{array}{l}
-\frac{\delta}{2}\left[(t-2)^{2}+3\right], \quad 0 \leqq t \leqq 1, \\
\delta(t-3), \quad 1 \leqq t \leqq 2 \\
-\delta, \quad 2 \leqq t \leqq 3
\end{array}\right.
$$




$$
\bar{x}(t)=\left\{\begin{array}{l}
1+t-\delta\left[\frac{3 t}{2}+\frac{1}{6}(t-2)^{3}+\frac{4}{3}\right], \quad 0 \leqq t \leqq 1, \\
\frac{3}{2}+\frac{t^{2}}{2}-\delta\left[4+\frac{3}{4}(t-1)^{2}+\frac{1}{24}(t-3)^{4}+\frac{4}{3}(t-1)-\frac{1}{2}(t-3)^{2}\right], \quad 1 \leqq t \leqq 2, \\
-\delta\left[\frac{547}{120}+5(t-2)+\frac{3}{2}(t-2)^{2}+\frac{1}{4}(t-2)^{3}-\frac{1}{6}(t-4)^{3}+\frac{1}{120}(t-4)^{5}\right],
\end{array}\right.
$$

$$
J(\bar{u})=\frac{329}{60} \delta^{2} \quad \text { where } \delta=\frac{185}{329} .
$$

The matrix valued function $\left[\Pi^{N}(t)\right], 0 \leqq t \leqq 3$, was computed as indicated in $\S 5.2$. The suboptimal trajectories $\hat{x}^{N}(t)$ were obtained solving $(6.1)$ with $u(t)=\hat{u}^{N}(t), \hat{u}^{N}(t)$ given by $(5.15)$, by a modified Runge-Kutta procedure. Then $\hat{u}^{N}(t)$ was computed from (5.15).

The numerical results we obtained are presented in Tables 6.1 and 6.2. We observe that the error $\left|\hat{u}^{N}(t)-\bar{u}(t)\right|$ is larger around $t=1$ and $t=2$ compared to other points in $[0,3]$ because there $\bar{u}(t)$ has jumps in the derivative whereas $\hat{u}^{N}(t)$ is continuously differentiable on $[0,3]$. In Table 6.2 we didn't include the values for $t=0$ because always $\hat{x}^{N}(0)=\bar{x}(0)$ for our algorithm.

6.2. Two examples for the infinite time horizon problem. For the examples equation (5.18) was solved using the Newton-Kleinman algorithm as presented in [35], for instance. The Lyapunov matrix equation which has to be solved in each step of this algorithm was solved using the quadratically convergent procedure given by R. A. Smith [40] (see also [35, p. 297]). The suboptimal trajectories $\hat{x}^{N}(t)$ and controls $\hat{u}^{N}(t)$ were calculated as for the example in $\S 6.1$ using (5.19). The two examples were already considered in [8] where the approximation was done based on the spline algorithm developed in [7].

\begin{tabular}{|c|c|c|c|c|}
\hline$\hat{u}$ & $\hat{u}^{4}(t)$ & $\hat{u}^{8}(t)$ & $\hat{u}^{16}(t)$ & $\bar{u}(t)$ \\
\hline 0 & -1.9694 & -1.9676 & -1.9679 & -1.9681 \\
\hline 0.25 & -1.7049 & -1.7049 & -1.7043 & -1.7045 \\
\hline 0.5 & -1.4740 & -1.4758 & -1.4760 & -1.4761 \\
\hline 0.75 & -1.2817 & -1.2824 & -1.2828 & -1.2828 \\
\hline 1.0 & -1.1267 & -1.1252 & -1.1250 & -1.1246 \\
\hline 1.25 & -0.9882 & -0.9832 & -0.9846 & -0.9840 \\
\hline 1.5 & -0.8410 & -0.8448 & -0.8445 & -0.8435 \\
\hline 1.75 & -0.6922 & -0.7002 & -0.7050 & -0.7029 \\
\hline 2.0 & -0.5885 & -0.5769 & -0.5704 & -0.5623 \\
\hline 2.25 & -0.5572 & -0.5611 & -0.5623 & \\
\hline 2.5 & -0.5620 & -0.5623 & -0.5623 & \\
\hline 2.75 & -0.5623 & -0.5623 & -0.5623 & \\
\hline 3.0 & -0.5621 & -0.5622 & -0.5623 & -0.5623 \\
\hline$J(\hat{u})$ & 1.7338 & 1.7338 & 1.7338 & 1.7338 \\
\hline
\end{tabular}

TABLE 6.1 
TABLE 6.2

\begin{tabular}{lllll}
\hline \multicolumn{1}{c}{$\hat{x}$} & $\hat{x}^{4}(t)$ & $\hat{x}^{8}(t)$ & $\hat{x}^{16}(t)$ & $\bar{x}(t)$ \\
\hline 0.25 & 0.7914 & 0.7916 & 0.7916 & 0.7917 \\
0.5 & 0.6448 & 0.6448 & 0.6448 & 0.6448 \\
0.75 & 0.5511 & 0.5506 & 0.5507 & 0.5507 \\
1.0 & 0.5007 & 0.5005 & 0.5005 & 0.5005 \\
1.25 & 0.4589 & 0.4593 & 0.4595 & 0.4595 \\
1.5 & 0.4083 & 0.4096 & 0.4094 & 0.4094 \\
1.75 & 0.3655 & 0.3642 & 0.3646 & 0.3646 \\
2.0 & 0.3375 & 0.3372 & 0.3371 & 0.3370 \\
2.25 & 0.3159 & 0.3167 & 0.3168 & 0.3168 \\
2.5 & 0.2845 & 0.2848 & 0.2848 & 0.2849 \\
2.75 & 0.2403 & 0.2407 & 0.2408 & 0.2408 \\
3.0 & 0.1874 & 0.1874 & 0.1874 & 0.1874 \\
\hline
\end{tabular}

The first example is Example 4.1 in [8] and considers the minimization of

$$
J(u)=\int_{0}^{\infty}\left[x(t)^{2}+u(t)^{2}\right] d t
$$

subject to

$$
\begin{aligned}
& \dot{x}(t)=x(t)+x(t-1)+u(t), \quad t \geqq 0, \\
& \phi^{0}=0, \quad \phi^{1}(t)=\sin \pi t, \quad-1 \leqq t \leqq 0 .
\end{aligned}
$$

In this case we have $n=p=1, A_{0}=A_{1}=B_{0}=C_{0}=R=1$. In Table 6.3 we give the values for $J\left(\hat{u}^{N}\right)$ and the optimal costs $J^{N}=\left\langle\Pi^{N} p^{N}\left(\phi^{0}, \phi^{1}\right), p^{N}\left(\phi^{0}, \phi^{1}\right)\right\rangle$ for the approximating systems $\left(\Sigma^{N}\right)$ with cost functional (5.16) and the corresponding values obtained in [8].

In Table 6.4 we show the values of $\Pi_{0}^{N}, \Pi_{1 N}^{N}$ and for $\Pi_{0}^{N}$ as obtained in [8]. Since range $\Pi \subset \operatorname{dom} A^{*}$ (cf. (3.15)), we have $A_{1}^{T} \Pi_{00} \phi^{0}=\left(\Pi_{10} \phi^{0}\right)(-1)$ for all $\phi^{0} \in \mathbb{R}^{n}$. Therefore in case of this example we should have

$$
\Pi_{0}^{N}-\Pi_{1 N}^{N} \rightarrow 0 \text { as } N \rightarrow \infty .
$$

In Table 6.5 we give the values for $\Pi_{1}^{N}(\tau)$, which governs the distributed feedback in

TABLE 6.3

\begin{tabular}{rcccc}
\hline$N$ & $J\left(\hat{u}^{N}\right)$ & $J^{N}$ & $J\left(\hat{u}^{N}\right),[8]$ & $J^{N},[8]$ \\
\hline 4 & 0.321439 & 0.321430 & 0.3272 & 0.2484 \\
8 & 0.321439 & 0.321432 & 0.3271 & 0.3027 \\
16 & 0.321439 & 0.321430 & 0.3272 & 0.3163 \\
\hline
\end{tabular}

TABLE 6.4

\begin{tabular}{rlll}
\hline$N$ & \multicolumn{1}{c}{$\Pi_{0}^{N}$} & $\Pi_{1 N}^{N}$ & $\Pi_{0}^{N},[8]$ \\
\hline 4 & 2.80886 & 2.77538 & 2.7940 \\
8 & 2.809328 & 2.80096 & 2.8054 \\
16 & 2.809390 & 2.80729 & 2.8084 \\
32 & 2.809396 & 2.80887 & 2.8091 \\
\hline
\end{tabular}


TABLE 6.5

\begin{tabular}{|c|c|c|c|c|}
\hline$J$ & $\Pi_{1}^{4}\left(-\frac{j}{32}\right)$ & $\Pi_{1}^{8}\left(-\frac{j}{32}\right)$ & $\Pi_{1}^{16}\left(-\frac{j}{32}\right)$ & $\Pi_{1}^{32}\left(-\frac{j}{32}\right)$ \\
\hline 0 & 0.63598 & 0.63683 & 0.63694 & 0.63696 \\
\hline 1 & - & - & - & 0.66132 \\
\hline 2 & - & - & 0.68698 & 0.68764 \\
\hline 3 & - & - & - & 0.71558 \\
\hline 4 & - & 0.74240 & 0.74512 & 0.74553 \\
\hline 5 & - & - & - & 0.77722 \\
\hline 6 & - & - & 0.81040 & 0.81114 \\
\hline 7 & - & - & - & 0.84695 \\
\hline 8 & 0.87064 & 0.88269 & 0.88474 & 0.88517 \\
\hline 9 & - & - & - & 0.92547 \\
\hline 10 & - & - & 0.96750 & 0.96839 \\
\hline 11 & - & - & - & 1.01361 \\
\hline 12 & - & 1.05757 & 1.06113 & 1.06165 \\
\hline 13 & - & - & - & 1.11225 \\
\hline 14 & - & - & 1.16491 & 1.16591 \\
\hline 15 & - & - & - & 1.22238 \\
\hline 16 & 1.26664 & 1.27879 & 1.28151 & 1.28218 \\
\hline 17 & - & - & - & 1.34508 \\
\hline 18 & - & - & 1.41048 & 1.41162 \\
\hline 19 & - & - & - & 1.48157 \\
\hline 20 & - & 1.54972 & 1.55463 & 1.55547 \\
\hline 21 & - & - & - & 1.63314 \\
\hline 22 & - & - & 1.71381 & 1.71512 \\
\hline 23 & - & - & - & 1.80125 \\
\hline 24 & 1.86588 & 1.88693 & 1.89104 & 1.89209 \\
\hline 25 & - & - & - & 1.98748 \\
\hline 26 & - & - & 2.08649 & 2.08802 \\
\hline 27 & - & - & - & 2.19358 \\
\hline 28 & - & 2.29692 & 2.30348 & 2.30477 \\
\hline 29 & - & - & - & 2.42147 \\
\hline 30 & - & - & 2.54253 & 2.54432 \\
\hline 31 & - & - & - & 2.67323 \\
\hline 32 & 2.77538 & 2.80096 & 2.80729 & 2.80887 \\
\hline
\end{tabular}

(6.3), at the knots $-j / N, j=0, \cdots, N$, for $N=4,8,16$ and 32 . We clearly see that $\Pi_{1}^{N}(\tau)$ converges uniformly on $-1 \leqq \tau \leqq 0$ as $N \rightarrow \infty$. Note, that $\Pi_{1}^{N}(\tau)$ is a continuous piecewise linear function on $[-1,0]$ with knots at $-j / N, j=0, \cdots, N$.

In Tables 6.6 and 6.7 we present the values for $\hat{u}^{N}(t)$ on $0 \leqq t \leqq 4$ and for $\hat{x}^{N}(t)$ on $0 \leqq t \leqq 3$, respectively.

The results of this example show a significant improvement in the qualitative behavior of our spline scheme compared to the scheme presented in [8]. In both schemes $\Pi_{1}^{N}(\tau)$ is a continuous piecewise linear function on $[-1,0]$. But in [8] this function is increasingly oscillatory with increasing $N$ (compare Figs. 4.1-4.4 in [8]), whereas in our scheme $\Pi_{1}^{N}(\tau)$ is strictly monotone and obviously converging in the supremum norm. This property of our scheme becomes very important if one wants to implement the approximating feedback law in a real system. Our scheme seems also to be more accurate as far as approximation of $\Pi_{00}$ by $\Pi_{0}^{N}$ and of $J(\bar{u})$ by $J^{N}\left(\hat{u}^{N}\right)$ or $J^{N}$ is concerned.

The next example is Example 4.2 in [8] and considers a simplified model for the Mach number control loop for the National Transonic Facility at NASA Langley 
TABLE 6.6

\begin{tabular}{llll}
\hline \multicolumn{1}{c}{$\hat{u}^{N}$} & $\hat{u}^{4}(t)$ & $\hat{u}^{8}(t)$ & $\hat{u}^{16}(t)$ \\
\hline 0 & & & \\
\hline 0.25 & 0.86836 & 0.86817 & 0.86816 \\
0.5 & 0.49650 & 0.64891 & 0.64891 \\
0.75 & 0.35400 & 0.36400 & 0.49658 \\
1.0 & 0.24627 & 0.24618 & 0.36401 \\
1.25 & 0.16154 & 0.16146 & 0.16146 \\
1.5 & 0.10999 & 0.10993 & 0.10993 \\
1.75 & 0.08024 & 0.08021 & 0.08021 \\
2.0 & 0.06015 & 0.06015 & 0.06015 \\
2.25 & 0.04348 & 0.04347 & 0.04347 \\
2.5 & 0.02983 & 0.02982 & 0.02982 \\
2.75 & 0.01996 & 0.01995 & 0.01995 \\
3.0 & 0.01373 & 0.01372 & 0.01372 \\
3.25 & 0.00991 & 0.00991 & 0.00991 \\
3.5 & 0.00729 & 0.00729 & 0.00729 \\
3.75 & 0.00524 & 0.00523 & 0.00523 \\
4.0 & 0.00362 & 0.00362 & 0.00362 \\
\hline
\end{tabular}

TABLE 6.7

\begin{tabular}{|c|c|c|c|}
\hline$\hat{x}$ & $\hat{x}^{4}(t)$ & $\hat{x}^{8}(t)$ & $\hat{x}^{16}(t)$ \\
\hline 0.25 & 0.11259 & 0.11258 & 0.11258 \\
\hline 0.5 & 0.05332 & 0.05331 & 0.05332 \\
\hline 0.75 & -0.06628 & -0.06626 & -0.06626 \\
\hline 1.0 & -0.10850 & -0.10846 & -0.10846 \\
\hline 1.25 & -0.06160 & -0.06158 & -0.06158 \\
\hline 1.5 & -0.01397 & -0.01397 & -0.01397 \\
\hline 1.75 & 0.00753 & 0.00752 & 0.00752 \\
\hline 2.0 & 0.00178 & 0.00178 & 0.00178 \\
\hline 2.25 & -0.00784 & -0.00784 & -0.00784 \\
\hline 2.5 & -0.01030 & -0.01029 & -0.01029 \\
\hline 2.75 & -0.00646 & -0.00646 & -0.00646 \\
\hline 3.0 & -0.00178 & -0.00178 & -0.00178 \\
\hline
\end{tabular}

Research Center. For details see [2] or [8]. The problem is to minimize

$$
J(u)=\int_{0}^{\infty}\left[x^{T}(t) C_{0}^{T} C_{0} x(t)+u^{2}(t)\right] d t
$$

subject to

$$
\begin{aligned}
\dot{x}(t) & =\left(\begin{array}{ccc}
-a & 0 & 0 \\
0 & 0 & 1 \\
0 & -\omega^{2} & -2 \xi \omega
\end{array}\right) x(t)+\left(\begin{array}{ccc}
0 & k a & 0 \\
0 & 0 & 0 \\
0 & 0 & 0
\end{array}\right) x(t-0.33)+\left(\begin{array}{c}
0 \\
0 \\
\omega^{2}
\end{array}\right) u(t), \quad t \geqq 0, \\
\phi^{0} & =\operatorname{col}(-0.1,8.547,0) \equiv \phi^{1}(t), \quad-0.33 \leqq t \leqq 0 .
\end{aligned}
$$


We have $C_{0}=(100,0,0), n=3, p=1, k=-0.0117, \xi=0.8, \omega=0.6$ and $1 / a=1.964$. Because of the simple structure of this problem it is possible to calculate the true solution following an idea contained in [29]. If we put for $t \geqq 0, h=0.33$

$$
y_{1}(t)=x_{1}(t+h), \quad y_{2}(t)=x_{2}(t), \quad y_{3}(t)=x_{3}(t),
$$

we obtain by a simple calculation

$$
\frac{d}{d t}\left(\begin{array}{l}
y_{1}(t) \\
y_{2}(t) \\
y_{3}(t)
\end{array}\right)=\left(\begin{array}{ccc}
-a & k a & 0 \\
0 & 0 & 1 \\
0 & -\omega^{2} & -2 \xi \omega
\end{array}\right)\left(\begin{array}{l}
y_{1}(t) \\
y_{2}(t) \\
y_{3}(t)
\end{array}\right)+\left(\begin{array}{c}
0 \\
0 \\
\omega^{2}
\end{array}\right) u(t) .
$$

The cost functional takes the form

$$
J(u)=10^{4} \int_{0}^{h} x_{1}(t)^{2} d t+\int_{0}^{\infty}\left[10^{4} y_{1}(t)^{2}+u(t)^{2}\right] d t,
$$

where

$$
x_{1}(t)=e^{-a t} \phi_{1}^{0}+a k \int_{0}^{t} e^{-a(t-\tau)} \phi_{2}^{1}(\tau-h) d \tau, \quad 0 \leqq t \leqq h
$$

is not dependent on $u(t)$ on the interval $[0, h]$. Therefore minimizing $J(u)$ subject to (6.2) is equivalent to minimizing

$$
\tilde{J}(u)=\int_{0}^{\infty}\left[10^{4} y_{1}(t)^{2}+u(t)^{2}\right] d t
$$

subject to (6.3) with initial data

$$
y_{1}(0)=x_{1}(h), \quad y_{2}(0)=x_{2}(0), \quad y_{3}(0)=x_{3}(0) .
$$

The solution of the latter problem is given by the feedback law

$$
\bar{u}(t)=-\left(0,0, \omega^{2}\right) \tilde{\Pi}_{0} \bar{y}(t),
$$

where $\bar{y}(t)$ is the solution of (6.3) with $u(t)=\bar{u}(t)$ and initial data (6.6). $\tilde{\Pi}_{0}$ is the solution of the algebraic Riccati equation

$$
\tilde{A}^{T} \tilde{\Pi}_{0}+\tilde{\Pi}_{0} \tilde{A}-\tilde{\Pi}_{0} B_{0} B_{0}^{T} \tilde{\Pi}_{0}+C_{0}^{T} C_{0}=0,
$$

where $\tilde{A}$ is the system matrix in (6.5) and $B_{0}, C_{0}$ are the same as for (6.2).

Equation (6.7) was solved numerically to give

$$
\tilde{\Pi}_{0}=\left(\begin{array}{rrr}
8220.51099 & -11.61086 & -1.12107 \\
-11.61086 & 0.01851 & 0.00186 \\
-1.12107 & 0.00186 & 0.00019
\end{array}\right) \text {. }
$$

The optimal costs for the original problem are given by (see (6.4))

$$
\begin{aligned}
J(\bar{u}) & =\tilde{J}(\bar{u})+10^{4} \int_{0}^{h} \bar{x}_{1}(t)^{2} d t \\
& =\bar{y}^{T}(0) \tilde{\Pi}_{0} \bar{y}(0)+10^{4} \int_{0}^{h} \bar{x}_{1}(t)^{2} d t .
\end{aligned}
$$

Using (6.5) it is easy to calculate $J(\bar{u})$. In Table 6.8 we give the values for $J(\bar{u}), J\left(\hat{u}^{N}\right)$ and $J^{N}=\left\langle\Pi^{N} p^{N}\left(\phi^{0}, \phi^{1}\right), p^{N}\left(\phi^{0}, \phi^{1}\right)\right\rangle$ and the values available in [8]. 
TABLE 6.8

\begin{tabular}{rcccc}
\hline$N$ & $J\left(\hat{u}^{N}\right)$ & $J^{N}$ & $J\left(\hat{u}^{N}\right),[8]$ & $J^{N},[8]$ \\
\hline 4 & 136.39587 & 136.40499 & 136.7354 & 138.7345 \\
8 & 136.40094 & 136.40509 & 136.7354 & 138.7624 \\
16 & 136.40250 & 136.40521 & - & - \\
\hline$J(\bar{u})$ & \multicolumn{5}{c}{136.40490} \\
\hline
\end{tabular}

Computing $J(\bar{u})$ for general initial data $\left(\phi^{0}, \phi^{1}\right)$ by using $(6.8),(6.6)$ and (6.5) and comparing the result with

$$
J(\bar{u})=\left(\phi^{0}\right)^{T} \Pi_{00} \phi^{0}+2\left(\phi^{0}\right)^{T} \Pi_{01} \phi^{1}+\left\langle\phi^{1}, \quad \Pi_{11} \phi^{1}\right\rangle_{L^{2}},
$$

we immediately obtain an explicit representation of $\Pi$ :

$$
\begin{gathered}
\Pi_{00}=\left(\begin{array}{ccc}
e^{-a h} & 0 & 0 \\
0 & 1 & 0 \\
0 & 0 & 1
\end{array}\right) \tilde{\Pi}_{0}\left(\begin{array}{ccc}
e^{-a h} & 0 & 0 \\
0 & 1 & 0 \\
0 & 0 & 1
\end{array}\right)+10^{4} \frac{1-e^{-2 a h}}{2 a}\left(\begin{array}{lll}
1 & 0 & 0 \\
0 & 0 & 0 \\
0 & 0 & 0
\end{array}\right), \\
\Pi_{01} \phi^{1}=a k\left(\begin{array}{ccc}
e^{-a h} & 0 & 0 \\
0 & 1 & 0 \\
0 & 0 & 1
\end{array}\right) \tilde{\Pi}_{0}\left(\begin{array}{lll}
0 & 1 & 0 \\
0 & 0 & 0 \\
0 & 0 & 0
\end{array}\right) \int_{-h}^{0} e^{a \tau} \phi^{1}(\tau) d \tau \\
+10^{4} k e^{-a h}\left(\begin{array}{lll}
0 & 1 & 0 \\
0 & 0 & 0 \\
0 & 0 & 0
\end{array}\right) \int_{-h}^{0} \frac{e^{-a \tau}-e^{a \tau}}{2} \phi^{1}(\tau) d \tau
\end{gathered}
$$

or, equivalently, $\left(\Pi_{01}^{*} \phi^{0}\right)(\theta)=\Pi_{1}(\theta) \phi^{0}$ with

$$
\Pi_{1}(\theta)=a k\left(\begin{array}{lll}
0 & 0 & 0 \\
1 & 0 & 0 \\
0 & 0 & 0
\end{array}\right) \tilde{\Pi}_{0}\left(\begin{array}{ccc}
e^{-a h} & 0 & 0 \\
0 & 1 & 0 \\
0 & 0 & 1
\end{array}\right) e^{a \theta}+10^{4} k e^{-a h}\left(\begin{array}{ccc}
0 & 0 & 0 \\
1 & 0 & 0 \\
0 & 0 & 0
\end{array}\right) \frac{e^{-a \theta}-e^{a \theta}}{2},
$$

$-h \leqq \theta \leqq 0$, and

$$
\begin{aligned}
\left(\Pi_{11} \phi^{1}\right)(\theta)=a^{2} k^{2} e^{a \theta}\left(\begin{array}{lll}
0 & 0 & 0 \\
1 & 0 & 0 \\
0 & 0 & 0
\end{array}\right) \tilde{\Pi}_{0}\left(\begin{array}{lll}
0 & 1 & 0 \\
0 & 0 & 0 \\
0 & 0 & 0
\end{array}\right) \int_{-h}^{0} e^{a \tau} \phi^{1}(\tau) d \tau \\
+a k^{2}\left(\begin{array}{lll}
0 & 0 & 0 \\
0 & 1 & 0 \\
0 & 0 & 0
\end{array}\right) \int_{-h}^{0} \frac{e^{-a|\tau-\theta|}-e^{a(\tau+\theta)}}{2} \phi^{1}(\tau) d \tau, \quad-h \leqq \theta \leqq 0 .
\end{aligned}
$$

In Table 6.9 we present the values for $\Pi_{0}^{N}, \Pi_{0}^{N}$ as computed in [8] and $\Pi_{00}$, whereas in Table 6.10 we give the values for the second row of $\Pi_{1}^{N}(-j h / 4)$ and $\Pi_{1}(-j h / 4)$ for $j=0, \cdots, 4$. The other rows of these matrices are always zero. Again, our scheme is more accurate compared to the scheme in [8] and $\Pi_{1}^{N}(\theta)$ converges uniformly on $[-h, 0]$ to $\Pi_{1}(\theta)^{T}$. 
TABLE 6.9

\begin{tabular}{|c|c|c|c|c|c|c|c|}
\hline$N$ & & $\Pi_{0}^{N}$ & & & & $\Pi_{0}^{N},[8]$ & \\
\hline \multirow{3}{*}{4} & /8677.02417 & -9.81502 & $-0.94768)$ & & 8676.9237 & -9.8164 & $-0.9477)$ \\
\hline & -9.81502 & 0.01851 & 0.00186 & & -9.8164 & 0.0185 & 0.0019 \\
\hline & -0.94768 & 0.00186 & 0.00019 & & -0.9477 & 0.0019 & 0.0002 \\
\hline \multirow{3}{*}{8} & 8677.02698 & -9.81505 & -0.94768 & & 8676.9829 & -9.8154 & $-0.9477)$ \\
\hline & -9.81505 & 0.01851 & 0.00186 & & -9.8154 & 0.0185 & 0.0019 \\
\hline & -0.94768 & 0.00186 & $0.00019 /$ & & -0.9477 & 0.0019 & $0.0002)$ \\
\hline \multirow{3}{*}{16} & 8677.03516 & -9.81506 & -0.94768 & & & & \\
\hline & -9.81506 & 0.01851 & 0.00186 & & & - & \\
\hline & -0.94768 & 0.00186 & 0.00019 & & & & \\
\hline \multirow{3}{*}{$\Pi_{00}$} & & & /8677.02405 & -9.81505 & -0.94768 & & \\
\hline & & & -9.81505 & 0.01851 & 0.00186 & & \\
\hline & & & -0.94768 & 0.00186 & 0.00019 & & \\
\hline
\end{tabular}

TABLE 6.10

\begin{tabular}{|c|c|c|c|}
\hline$j$ & & $\Pi_{1}^{4}\left(-\frac{j h}{4}\right)$ & \\
\hline 0 & -41.39697 & 0.06916 & 0.00668 \\
\hline 1 & -43.83789 & 0.06652 & 0.00640 \\
\hline 2 & -46.37943 & 0.06334 & 0.00613 \\
\hline 3 & -48.97898 & 0.06118 & 0.00590 \\
\hline 4 & -51.69006 & 0.05828 & 0.00563 \\
\hline$j$ & & $\Pi_{1}^{8}\left(-\frac{j h}{4}\right)$ & \\
\hline 0 & -41.39721 & 0.06917 & 0.00668 \\
\hline 1 & -43.84998 & 0.06626 & 0.00640 \\
\hline 2 & -46.38019 & 0.06355 & 0.00614 \\
\hline 3 & -48.99226 & 0.06093 & 0.00588 \\
\hline 4 & -51.69080 & 0.05843 & 0.00564 \\
\hline$j$ & & $\Pi_{1}^{16}\left(-\frac{j h}{4}\right)$ & \\
\hline 0 & -41.39727 & 0.06917 & 0.00668 \\
\hline 1 & -43.85012 & 0.06631 & 0.00640 \\
\hline 2 & -46.38036 & 0.06358 & 0.00614 \\
\hline 3 & -48.99246 & 0.06097 & 0.00589 \\
\hline 4 & -51.69102 & 0.05846 & 0.00564 \\
\hline$j$ & & $\Pi_{1}\left(-\frac{j h}{4}\right)$ & \\
\hline 0 & -41.39721 & 0.06917 & 0.00668 \\
\hline 1 & -43.85008 & 0.06632 & 0.00641 \\
\hline 2 & -46.38034 & 0.06360 & 0.00614 \\
\hline 3 & -48.99246 & 0.06098 & 0.00589 \\
\hline 4 & -51.69103 & 0.05847 & 0.00565 \\
\hline
\end{tabular}


Acknowledgment. F. Kappel acknowledges the hospitality provided by the members of the Forschungsschwerpunkt Dynamische Systeme, University of Bremen.

\section{REFERENCES}

[1] J. H. Ahlberg, E. N. Nilsen And J. C. Walsh, The Theory of Splines and Their Applications, Academic Press, New York, 1967.

[2] E. S. Armstrong AND J. S. TRIPP, An application of multivariable design techniques to the control of the National Transonic Facility, NASA Technical Paper 1887, NASA Langley Research Center, Hampton, VA, 1981.

[3] H. T. BANKS, Identification of nonlinear delay systems using spline methods, Proc. Internat. Conf. on Nonlinear Phenomena in Math. Sci., Arlington, TX, June 1980.

[4] H. T. BANks AND J. A. Burns, Hereditary control problems: Numerical methods based on averaging approximation, this Journal, 16 (1978), pp. 169-208.

[5] H. T. BANKS, J. A. Burns, E. M. ClifF AND P. R. THRIFT, Numerical solutions of hereditary control problems via an approximation technique, Brown University, LCDS Technical Report 15-6, Providence, RI, 1975.

[6] H. T. BANKS AND P. L. DANIEL, Parameter estimation of nonlinear nonautonomous distributed systems, Proc. 20th IEEE Conf. Decision and Control, San Diego, CA, December 1981, pp. 228-232.

[7] H. T. BANKS AND F. KAPPEL, Spline approximation for functional differential equations, J. Differential Equations, 34 (1979), pp. 496-522.

[8] H. T. BAnks, G. I. Rosen AND K. ITO, A spline based technique for computing Riccati operators and feedback controls in regulator problems for delay equations, SIAM J. Sci. Statist. Comput., 5 (1984), pp. 830-855.

[9] A. Bensoussan, M. C. Delfour And S. K. Mitter, The linear quadratic optimal control problem for infinite dimensional systems over an infinite horizon; survey and examples, Electronic Systems Laboratory Reports, Massachusetts Inst. Technology, Cambridge, MA, 1977.

[10] C. BERNIER AND A. MANITIUS, On semigroups in $\mathbb{R}^{n} \times L^{p}$ corresponding to differential equations with delays, Canad. J. Math., 30 (1978), pp. 897-914.

[11] K. BoEHMER, Spline-Funktionen, Theorie und Anwendungen, Teubner, Stuttgart, 1974.

[12] J. Borisovič AND A. S. Turbabin, On the Cauchy problem for linear nonhomogeneous differential equations with retarded argument, Soviet Math. Dokl., 10 (1969), pp. 401-405.

[13] J. A. Burns AND T. L. Herdman, Adjoint semigroup theory for a class of functional differential equations, SIAM J. Math. Anal., 5 (1976), pp. 729-745.

[14] R. F. Curtain And A. J. Pritchard, Infinite Dimensional Linear Systems Theory, Springer, Berlin, New York, 1978.

[15] R. DATKO, Unconstrained control problems with quadratic cost, this Journal, 11 (1973), pp. 32-52.

[16] M. C. Delfour, E. B. LEE AND A. MANitius, F-reduction of the operator Riccati equation, Automatica, 14 (1978), pp. 385-395.

[17] M. C. Delfour AND A. MANitius, The structural operator $F$ and its role in the theory of retarded systems, Part I: J. Math. Anal. Appl., 73 (1980), pp. 466-490; Part II: J. Math. Anal. Appl., 74 (1980), pp. 359-381.

[18] M. C. Delfour AND S. K. MitTer, Hereditary differential systems with constant delays I: General case, J. Differential Equations, 12 (1972), pp. 213-235.

[19] J. S. GiBSON, The Riccati integral equations for optimal control problems in Hilbert spaces, this Journal, 17 (1979), pp. 537-565.

[20] - Linear quadratic optimal control of hereditary differential systems: infinite dimensional Riccati equations and Numerical approximations, this Journal, 21 (1983), pp. 95-139.

[21] D. Gottlieb And S. A. Orszag, Numerical Analysis of Spectral Methods: Theory and Applications, CBMS-NSF Regional Conference Series in Applied Mathematics, Society for Industrial and Applied Mathematics, Philadelphia, PA, 1977.

[22] J. K. HALE, Theory of Functional Differential Equations, Springer, Berlin, New York, 1977.

[23] A. S. B. Holland And B. N. Sahney, The General Problem of Approximation and Spline Functions, Krieger, Huntington, New York, 1979.

[24] F. KAPPEL, Finite dimensional approximation of systems with infinite dimensional state space, in EQUADIFF 82, H. W. Knobloch and K. Schmitt, eds., Lecture Notes in Mathematics 1017, Springer, Berlin, 1983, pp. 287-299.

[25] F. KAPPEL AND G. PROPST, Approximation of feedback controls for delay systems using Legendre polynomials, Confer. Sem. Mat. Univ. Bari, 201 (1984), pp. 1-36. 
[26] F. KAPPEL AND D. SAlamon, Spline approximation for retarded systems and the Riccati equation, Institute for Mathematics, University of Graz, Preprint No. 42-1984, and Mathematics Research Center, University of Wisconsin, Technical Summary Report No. 2680, April 1984.

[27] K. Kunisch, Approximation schemes for the linear quadratic optimal control problem associated with delay equations, this Journal, 20 (1982), pp. 506-540.

[28] A. MANitius, Completeness and F-completeness of eigenfunctions associated with retarded functional differential equations, J. Differential Equations, 35 (1980), pp. 1-29.

[29] A. MANITIUS AND H. TRAN, Numerical simulation of a nonlinear feedback controller for a wind tunnel model involving a time delay, Optimal Control Appl. Meth., 7 (1986), pp. 19-39.

[30] R. H. MARTIN, JR., Nonlinear Operators and Differential Equations in Banach Spaces, John Wiley, New York, 1976.

[31] R. K. Miller, Linear Volterra integro-differential equations as semigroups, Funkcial. Ekvac., 17 (1974), pp. 39-55.

[32] A. PAZY, Semigroups of Linear Operators and Applications to Partial Differential Equations, Springer, Berlin, New York, 1983.

[33] L. PANDOLFI, Feedback stabilization of functional differential equations, Boll. Un. Mat. Ital., 12 (1975), pp. 626-635.

[34] G. Propst, Piecewise linear approximation for hereditary control problems, Institute for Mathematics, University of Graz, Technical Report No. 60-1985, Graz, 1985.

[35] D. L. Russell, Mathematics of Finite Dimensional Control Systems, Theory and Design, Marcel Dekker, New York, 1979.

[36] D. Salamon, On dynamic observation and state feedback for time delay systems, in Evolution Equations and Their Applications, F. Kappel and W. Schappacher, eds., Research Notes in Math., Pitman, London, 1982, pp. 202-219.

[37] - Control and Observation of Neutral Systems, Research Notes in Math., 91, Pitman, London, 1984.

[38] - Structure and stability of finite dimensional approximations for functional differential equations, University of Wisconsin-Madison, TSR\# 2586, 1983.

[39] M. H. Schultz, Spline Analysis, Prentice-Hall, Englewood Cliffs, NJ, 1973.

[40] R. A. SMith, Matrix equation $X A+B X=C$, SIAM J. Appl. Math., 16 (1968), pp. 198-201.

[41] J. ZABCZYK, Remarks on the algebraic Riccati equation in Hilbert space, Appl. Math. Optim., 2 (1976), pp. 251-258. 